\title{
Long-duration magnetic clouds: a comparison of analyses using torus- and cylinder-shaped flux rope models
}

\author{
K. Marubashi ${ }^{1, *}$ and R. P. Lepping ${ }^{2}$ \\ ${ }^{1}$ National Institute of Information and Communications Technology, Koganei, Tokyo 184-8795, Japan \\ ${ }^{2}$ Heliophysics Science Division, NASA-Goddard Space Flight Center, Greenbelt, MD 20771, USA \\ *now at: Korea Astronomy and Space Science Institute, Daejeon 305-348, Korea
}

Received: 4 March 2007 - Revised: 11 September 2007 - Accepted: 18 October 2007 - Published: 29 November 2007

\begin{abstract}
We identified 17 magnetic clouds (MCs) with durations longer than $30 \mathrm{~h}$, surveying the solar wind data obtained by the WIND and ACE spacecraft during 10 years from 1995 through 2004. Then, the magnetic field structures of these 17 MCs were analyzed by the technique of the least-squares fitting to force-free flux rope models. The analysis was made with both the cylinder and torus models when possible, and the results from the two models are compared. The torus model was used in order to approximate the curved portion of the MCs near the flanks of the MC loops. As a result, we classified the $17 \mathrm{MCs}$ into 4 groups. They are (1) $5 \mathrm{MC}$ events exhibiting magnetic field rotations through angles substantially larger than $180^{\circ}$ which can be interpreted only by the torus model; (2) 3 other MC events that can be interpreted only by the torus model as well, though the rotation angles of magnetic fields are less than $180^{\circ}$; (3) $3 \mathrm{MC}$ events for which similar geometries are obtained from both the torus and cylinder models; and (4) $6 \mathrm{MC}$ events for which the resultant geometries obtained from both models are substantially different from each other, even though the observed magnetic field variations can be interpreted by either of the torus model or the cylinder model. It is concluded that the $\mathrm{MC}$ events in the first and second groups correspond to those cases where the spacecraft traversed the MCs near the flanks of the MC loops, the difference between the two being attributed to the difference in distance between the torus axis and the spacecraft trajectory. The MC events in the third group are interpreted as the cases where the spacecraft traversed near the apexes of the MC loops. For the MC events in the fourth group, the real geometry cannot be determined from the model fitting technique alone. Though an attempt was made to determine which model is more plausible for each of the MCs in this group by comparing the characteristics of associated bidirectional electron heat flows, the results
\end{abstract}

Correspondence to: K. Marubashi

(k.marubashi@eos.ocn.ne.jp) were not very definitive. It was also found that the radii of the flux ropes obtained from the torus fitting tend to be generally smaller than those obtained from the cylinder fitting. This result raises a possible problem in estimating the magnetic flux and helicity carried away from the Sun by the MCs.

Keywords. Interplanetary physics (Interplanetary magnetic fields; Solar wind plasma)

\section{Introduction}

Coronal mass ejections (CMEs) launch plasma clouds from the solar atmosphere into interplanetary space which are now referred to as interplanetary coronal mass ejections (ICMEs). ICMEs are identified by several characteristic features in the interplanetary medium, as reviewed by Gosling (1990) and Neugebauer and Goldstein (1997). Solar wind signatures of ICMEs include the increase of $\mathrm{He}^{++}$(Hirshberg et al., 1972; Borrini et al., 1982), the abnormally low proton temperature (Gosling et al., 1973; Richardson and Cane, 1995), the bidirectional electron heat flow (Gosling et al., 1987), and the magnetic cloud (Burlaga et al., 1981; Marubashi, 2000; Lepping et al., 2006), though the regions of these ICME signatures do not necessarily coincide with each other.

An interplanetary magnetic cloud (MC) can occupy the whole body of an ICME or a significant part of it. The global configurations of MCs in interplanetary space and their internal magnetic structures provide important information about the connection between ICMEs and their causative CMEs, which is crucial for developing models for the generation mechanism of CMEs. MCs are also important to magnetosphere dynamics, because they usually carry strong southward magnetic fields (Zhao et al., 2001) that strongly drive geomagnetic activity (e.g. Dungey, 1961; Tsurutani and Gonzalez, 1997, and references therein).

Published by Copernicus Publications on behalf of the European Geosciences Union. 


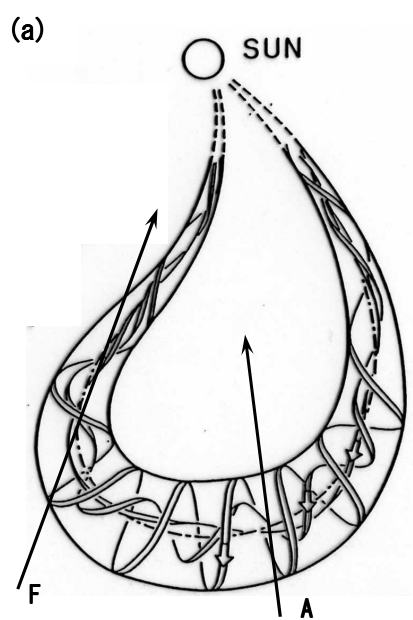

(b)

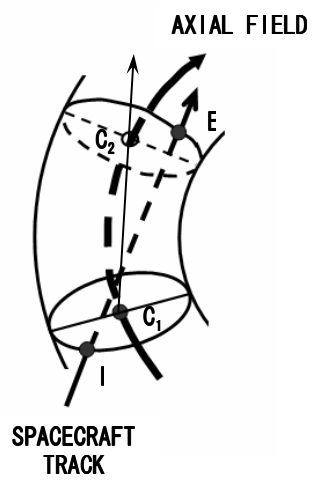

Fig. 1. (a) A schematic depicting a global configuration of an $\mathrm{MC}$ and two spacecraft passages: the apex passage (A), and the flank passage $(\mathrm{F})$. (b) A sketch of a torus-shaped flux rope representing the part of an MC for the flank passage. Provided that the spacecraft traverses the $\mathrm{MC}$ from point I through point $\mathrm{E}$, the vector from $\mathrm{C}_{1}$ to $\mathrm{C}_{2}$ is taken as the equivalent local direction of the $\mathrm{MC}$ axis, where $\mathrm{C}_{1}$ and $\mathrm{C}_{2}$ are the centers of two cross-sectional circles passing through points I and $\mathrm{E}$, respectively.

An MC's global configuration is proposed to be a loop extending from the Sun with both legs rooted on the Sun (Marubashi, 1989, 1997; Burlaga et al., 1990), and the internal magnetic field structure to be a magnetic flux rope, as is depicted in Fig. 1a (Goldstein, 1983; Marubashi, 1986; Burlaga, 1988; Lepping et al., 1990; Bothmer and Schwenn, 1998). When a spacecraft encounters an MC, the magnetic field vectors generally exhibit the rotation characteristic of the flux rope structure. The geometry of an MC at the location of the encounter with the spacecraft can be determined by comparing the observed magnetic field variations with those calculated from the magnetic flux rope model. When the spacecraft traversed near the apex of the loop, as is shown by passage A, the MC's geometry can be analyzed by using a cylinder model, because the local geometry can be taken approximately to be that of a straight cylinder. When the spacecraft traversed MCs near the flank of the loop, as is shown by passage $\mathrm{F}$, however, curvature of the MCs must be taken into consideration to explain the observed magnetic field variations. The curvature effects can be taken into account by using a torus-shaped flux rope model. Figure $1 \mathrm{~b}$ shows how a torus shape is applied as a proxy of the curved portion of the MC loop. Here, points I and E are the entry and exit of a spacecraft through the MC, respectively, and the torus shape is assumed only for the region bounded by two cross sections passing I and E. We can define the local orientation of the torus MC to be the orientation of a vector connecting $\mathrm{C}_{1}$ and $\mathrm{C}_{2}$, the centers of the two cross-sectional circles. It should be emphasized that the torus-shaped flux rope model is applied only for the limited portion of the MC loop, and it is not intended to imply that the entire torus could represent the global flux rope.

While many studies have been made to analyze an MC's geometry with cylindrical flux rope models (Lepping et al., 1990; Bothmer and Schwenn, 1998; Shimazu and Marubashi, 2000; Mulligan et al., 1998, 2001; Lynch et al., 2003; Lepping et al., 2006), only a few studies were made with torus models (Marubashi, 1997, 2000; Romashets and Vandas, 2003), or with other different models (Vandas and Geranios, 2001; Vandas and Romashets, 2003). Judging from the proposed global configuration of MCs, there should be more encounters with MCs near the flank than identified thus far.

In this study, we attempted to identify in a systematic way as many MC events as possible that require considering the curvature effects of the global configuration. For this purpose, we examined the solar wind data obtained by the WIND and ACE spacecraft during 10 years, from 1995 through 2004, with special attention to identifying the MC events with durations longer than $30 \mathrm{~h}$, which corresponds to about two times the most frequently observed MCs (Lepping et al., 2006). The selection of long-duration events is based on an idea that the durations of spacecraft passage through MCs tend to be longer when traversed near the flanks of loop structures of MCs compared with those cases when traversed near the apex, as is expected from Fig. 1a. As a result, we identified 17 long-duration MCs. Then, we performed the model fitting analysis for these 17 events with both cylinder and torus models, when possible, and compared the results from the two different models. This comparison shows that the $17 \mathrm{MC}$ events are categorized into several different groups: such as (1) MCs which can be well interpreted only by the torus model, (2) MCs for which both the cylinder and torus models provide satisfactory fitting results with similar geometries, and (3) MCs for which the two models provide satisfactory fitting results but their geometries are substantially different from each other.

In the next section, we present the overview of the selected 17 MCs used in this investigation. Section 3 describes the two flux rope models, a cylinder model and a torus model, which are applied to the fitting analysis. In Sect. 4, we show the results of fitting with the two models for the selected 17 MCs, and attempt to determine which model is more satisfactory in interpreting each MC observation by an intercomparison between the two fitting results. Finally in Sect. 5, we summarize the results and give the possible impacts of the present study, especially for the torus-shaped MCs, on our understanding of the ICME physics.

\section{Event selection}

We surveyed solar wind data obtained from the WIND and ACE spacecraft during 10 years from 1995 through 2004, 
Table 1. Magnetic cloud information.

\begin{tabular}{|c|c|c|c|c|c|c|c|c|c|c|c|c|c|}
\hline \multirow{2}{*}{$\begin{array}{c}\text { EVENT } \\
\text { NO. }\end{array}$} & \multicolumn{3}{|c|}{ START } & \multirow{2}{*}{$\begin{array}{c}\text { END } \\
\text { DAY/TIME }\end{array}$} & \multirow{2}{*}{$\begin{array}{c}\Delta T^{\mathrm{a}} \\
\mathrm{HR}\end{array}$} & \multirow{2}{*}{$\begin{array}{l}\text { SHOCK(D) } \\
\text { DAY/TIME }\end{array}$} & \multirow{2}{*}{$\begin{array}{l}\text { SHOCK(I) } \\
\text { DAY/TIME }\end{array}$} & \multirow{2}{*}{$\begin{array}{c}\text { ROT. } \\
\text { ANGLE }^{\text {b }}\end{array}$} & \multirow{2}{*}{$\begin{array}{c}\text { AVERAGE } \\
\mathrm{Brms} / \mathrm{B}^{\mathrm{c}}\end{array}$} & \multirow{2}{*}{$\begin{array}{l}\text { AVERAGE } \\
\mathrm{He}^{++} / \mathrm{H}^{+}\end{array}$} & \multirow{2}{*}{$\begin{array}{c}\text { AVERAGE } \\
T_{p} / T_{e x}^{\mathrm{d}}\end{array}$} & \multirow{2}{*}{$\begin{array}{c}\text { BDE } \\
\text { D.PA }\end{array}$} & \multirow{2}{*}{$\begin{array}{c}\text { DATA } \\
\text { SOURCE }\end{array}$} \\
\hline & YR & MON & DAY/TIME & & & & & & & & & & \\
\hline 1 & 95 & 10 & 18/19:00 & 20/01:30 & 30.5 & 18/10:30 & $19 / 17: 51$ & 178 & 0.041 & 0.058 & 0.83 & $180^{\circ}$ & WIND \\
\hline 2 & 96 & 05 & $27 / 15$ - & $28 / 23-$ & 32 & - & - & -125 & 0.063 & - & 0.71 & $0^{\circ}$ & WIND \\
\hline 3 & 96 & 12 & $24 / 03-$ & 25/11:31 & 32.5 & - & - & 194 & 0.038 & 0.053 & 0.94 & $180^{\circ}$ & WIND \\
\hline 4 & 97 & 10 & $01 / 17-$ & $02 / 23: 51$ & 30.8 & 01/01:41 & - & -75 & 0.037 & 0.044 & 0.32 & $0^{\circ}$ & WIND \\
\hline 5 & 98 & 01 & 07/02:49 & $08 / 12-$ & 33.2 & 06/13:28 & - & -209 & 0.042 & 0.053 & 0.76 & $0^{\circ}$ & WIND \\
\hline 6 & 98 & 02 & $04 / 03-$ & $05 / 22-$ & 43 & - & - & -329 & 0.036 & 0.027 & 0.53 & $0^{\circ}$ & WIND \\
\hline 7 & 98 & 03 & $04 / 19-$ & $06 / 05-$ & 34 & 04/10:58 & - & -109 & 0.036 & 0.104 & 0.64 & UNI $0^{\circ}$ & $\mathrm{ACE}$ \\
\hline 8 & 98 & 05 & 02/11:48 & 03/21:08 & 33.3 & $01 / 21: 21$ & 03/16:59 & 59 & 0.033 & 0.130 & 0.10 & $180^{\circ}$ & $\mathrm{ACE}$ \\
\hline 9 & 98 & 06 & $24 / 13-$ & $25 / 22: 45$ & 33.8 & - & $25 / 15: 43$ & -180 & 0.023 & 0.049 & 0.42 & $0^{\circ}$ & $\mathrm{ACE}$ \\
\hline 10 & 98 & 08 & $20 / 09-$ & $21 / 20: 22$ & 35.4 & 19/18:40 & - & 175 & 0.031 & 0.049 & 1.39 & $180^{\circ}$ & WIND \\
\hline 11 & 98 & 11 & $08 / 18-$ & $10 / 01-$ & 31 & 08/04:21 & - & 286 & 0.027 & 0.065 & 0.39 & MIX & $\mathrm{ACE}$ \\
\hline 12 & 00 & 10 & 03/16:55 & $05 / 05-$ & 36.1 & 03/01:01 & $05 / 03: 29$ & 228 & 0.040 & 0.049 & 0.32 & $0^{\circ}$ & WIND \\
\hline 13 & 01 & 03 & $19 / 21-$ & $22 / 06-$ & 57 & $19 / 11: 33$ & - & -194 & 0.042 & 0.058 & 0.71 & $180^{\circ}$ & WIND \\
\hline 14 & 01 & 05 & $09 / 15-$ & $10 / 22: 43$ & 31.7 & - & - & 86 & 0.079 & 0.081 & 0.41 & $180^{\circ}$ & WIND \\
\hline 15 & 02 & 04 & $18 / 01-$ & $19 / 11-$ & 34 & 17/10:21 & 19/08:02 & 53 & 0.021 & 0.120 & 0.28 & MIX & ACE \\
\hline 16 & 03 & 10 & $22 / 17: 57$ & $24 / 02: 27$ & 32.5 & - & - & 12 & 0.028 & 0.090 & 0.15 & MIX & $\mathrm{ACE}$ \\
\hline 17 & 04 & 04 & $03 / 23: 53$ & $05 / 13: 30$ & 37.6 & 03/08:55 & - & -190 & 0.021 & 0.098 & 0.29 & MIX & $\mathrm{ACE}$ \\
\hline
\end{tabular}

a $\Delta T$ is the duration of the encounter with the magnetic cloud.

$\mathrm{b}$ The rotation angle of the magnetic field vector within the magnetic cloud projected on the Y-Z plane.

${ }^{\mathrm{c}}$ Brms is the combination of the RMS values of $\mathrm{Bx}, \mathrm{By}$, and $\mathrm{Bz}$ obtained from underlying high-resolution measurements.

${ }^{\mathrm{d}} T_{p}$ is the measured proton temperature and $T_{e x}$ is the proton temperature expected from the solar wind speed statistically.

e BDE D.PA is the dominant pitch angle within the associated bidirectional electron fluxes.

in search of the long-duration magnetic clouds. The solar wind plasma and field data used in this survey are from the Solar Wind Experiment (SWE) (Ogilvie et al., 1995) and the Magnetic Field Investigation (MFI) (Lepping et al., 1995) on the WIND spacecraft, and the Solar Wind Electron Proton Alpha Monitor (SWEPAM) (McComas et al., 1998) and the Magnetic Field Experiment (MAG) (Smith et al., 1998) on the Advanced Composition Explorer (ACE).

In order to make this survey as complete as possible, we first made plots of the magnetic field vectors, such as that shown in the bottom diagram of Fig. 2, for all the data period. Then, using the plots, we selected the long-duration MCs by the criteria: (1) the magnetic fields are relatively strong ( $\geq 10 \mathrm{nT})$; (2) the smooth rotations of magnetic field vectors are clear, being free from a significant interrupting interval; and (3) the durations of such intervals are longer than $30 \mathrm{~h}$. As a result of this survey, we identified $17 \mathrm{MCs}$ with long durations.

Table 1 lists the 17 long-duration MCs we identified and the specific characteristics of the MCs. The first 4 columns indicate the event identification No., the start and end times, and the durations of the MCs. When the boundaries of the MCs are identified by any discontinuous changes in the magnetic field and plasma parameters, the start and end times are shown in the unit of minutes, otherwise, they are given in the unit of hours. The next 2 columns show the arrival times of shocks associated with the MCs. Two kinds of shocks are shown in the table. One is the shock probably driven by the ICME relevant to each MC, and the other is an internal shock probably having overtaken the ICME from behind (Collier et al., 2007). The driven shocks are seen in 11 cases, and the internal shocks in 5 cases. The next 5 columns present five characteristic quantities related to the solar wind signatures which are generally used when identifying ICMEs: the magnetic field rotation, the degree of magnetic field fluctuations, the enhancement of $\mathrm{He}^{++}$abundance, the abnormally low proton temperature, and the bidirectional electron heat flux. Finally, the last column indicates the spacecraft providing the data source for the present analysis.

We briefly describe the characteristics of the MCs summarized in Table 1. Column 7 lists the angle of the magnetic field rotation in the plane perpendicular to the EarthSun line (YZ-plane). The rotation angles here are calculated using the magnetic field vectors averaged over the first $3 \mathrm{~h}$ and over the last $3 \mathrm{~h}$ of the MCs traversed by the spacecraft. We used 3-h averages to avoid errors in estimating the rotation angles due to possible sudden direction changes near the MC boundaries. Though this averaging may cause underestimation of the rotation angles, its effect is not very large. It should be pointed out that some of the MCs exhibit the magnetic field rotations through angles substantially exceeding $180^{\circ}$. Column 8 shows the degree of fluctuations in the magnetic field, defined by the ratio of standard deviations to the average intensity. Though we did not deduce typical values of the degree of fluctuations in the background solar wind, it will be seen later in many examples that this quantity is much reduced within MCs. Column 9 lists the values of the $\mathrm{He}^{++} / \mathrm{H}^{+}$number density ratio averaged within the MCs. A 
recent statistic study by Richardson and Cane (2004) showed that the enhanced ratio of $\mathrm{He}^{++} / \mathrm{H}^{+}(>0.06)$ is indicative of ICMEs. It should be noted here that the $\mathrm{He}^{++} / \mathrm{H}^{+}$ratios are seen to be generally enhanced within these MCs, compared to the surrounding regions, even though the averages are less than 0.06 , as will be seen later for several examples. Event 6 was the only exceptional case in that it exhibits no enhancement in this composition ratio. Column 10 shows the averages within the MCs of the ratio of observed proton temperature, $T_{p}$, to the proton temperature statistically expected from the solar wind speed, $T_{e x}$ (Lopez, 1987). It is seen that the proton temperature ratios are appreciably lower than those for the background solar wind, except for two cases (Events 3 and 10). Column 11 denotes the pitch angles of the dominant electron fluxes in the energy range $100-300 \mathrm{keV}$, taken by visual inspection of the plots from the WIND/3DP Plasma instrument (Lin et al., 1995) and from the ACE/SWEPAM phase 3 data plots. (The data are accessible through the websites http://sprg.ssl.berkeley.edu/ wind3dp/ and http://swepam-pub.lanl.gov/plots/ele/stea/, respectively.) Bidirectional electron fluxes are generally seen in association with the $17 \mathrm{MCs}$, of which the dominant pitch angles are easily identified. In some cases, however, the directions of dominant fluxes switched from $0^{\circ}$ to $180^{\circ}$ or vice versa during the $\mathrm{MC}$ intervals, and in one case the electron heat flux was unidirectional. These cases are indicated by marks, MIX and UNI, respectively. In summary, in most of the 17 MCs we see plasma and field signatures which are widely accepted to be characteristic of ICMEs, as was previously pointed out (Gosling, 1990; Neugebauer and Goldstein, 1997).

\section{Fitting with two flux rope models}

In examining the geometry of the MCs listed in Table 1, we apply a model fitting technique using two types of flux rope models, a cylinder model and a torus model. This section briefly describes each of these two models so that the meanings of model parameters can be understood. Appendix A and Appendix B give the expressions needed for calculations of magnetic fields and velocity variations which should be observed when the spacecraft traversed these models. The nonlinear least-squares fitting technique is described in Marubashi (2002).

\subsection{Cylinder model}

We use the constant- $\alpha$ force-free model, including a selfsimilar expansion, which was originally proposed by Farrugia et al. $(1992,1993)$, with a slight modification based on a proposal by Shimazu and Vandas (2002). In this model, the flux rope radius, $r$, the expansion velocity, $v$, and the magnetic field, $\boldsymbol{B}$, at time $t$ after the time of the first encounter with a spacecraft, are presented by the following expressions.
Here, the cylindrical coordinates, $\rho, \varphi, \zeta$, are used with the $\zeta$-axis along the axial magnetic field of the flux rope.

$$
\begin{aligned}
& r(t)=r_{0}(1+E t) \\
& \boldsymbol{v}_{\rho}(t)=\{E \rho /(1+E t)\} \boldsymbol{e}_{\rho}(\rho \leq r) \\
& \boldsymbol{B}(t)=B_{\varphi} \boldsymbol{e}_{\varphi}+B_{\zeta} \boldsymbol{e}_{\zeta} \\
& B_{\varphi}=s B_{0} J_{1}(\alpha \rho) /(1+E t)^{2} \\
& B_{\zeta}=B_{0} J_{0}(\alpha \rho) /(1+E t)^{2}
\end{aligned}
$$

Here $J_{0}$ and $J_{1}$ are Bessel functions of the first kind of order 0 and 1 , respectively, $B_{0}$ is the magnetic field intensity at the cylinder axis at time $t=0$, and $E$ is a parameter expressing the expansion rate. In the force-free field, the electric current flows parallel or anti-parallel to the magnetic field, and they correspond to $s=1$ and $s=-1$, respectively. This parameter also denotes the sign of magnetic field chirality, $s=1$ for righthanded and $s=-1$ for left-handed. In Eqs. (4) and (5), $\alpha$ is chosen so that $\alpha r$ gives the first zero of $J_{0}$ (i.e. $\alpha r \cong 2.405$ ). It should be noted here that $\alpha$ changes with time while it is constant spatially.

The expansion effect on $B_{\varphi}$ was proposed originally to be in proportion to $(1+E t)^{-1}$ (Farrugia et al., 1992). This dependency comes from the assumption that the expansion does not occur in the direction of the cylinder axis when a cylinder of infinite length is considered. Later, Shimazu and Vandas (2002) showed that the effect is given by Eq. (4) when the expansion along the cylinder axis is considered. The forcefree condition is maintained throughout the passage of $\mathrm{MC}$ by this modification.

The parameters of MCs to be determined by the model fitting are summarized below.

1. $U_{0}$ : the bulk flow velocity of the solar wind, or the speed of the MC at the center. The solar wind velocity variation within an MC is taken as the vector sum of the constant flow velocity $U_{0}$ and the expansion velocity given by Eq. (2).

2. $B_{0}$ and $r_{0}$ : the intensity of the magnetic field at the cylinder axis and the radius of the MC cylinder at time $t=0$, as described above.

3. $\theta_{a}, \phi_{a}$, and $p$ : the latitude and longitude angles of the cylinder axis and the impact parameter. The latitude and longitude angles are given in GSE coordinates. The impact parameter is given by the distance from the $\zeta$ axis to the spacecraft trajectory (assumed to be along the GSE $X$-axis) normalized by $r_{0}$. This quantity is defined to be measured along the vector product of two vectors: one parallel to the $\mathrm{X}$-axis and the other parallel to the $\zeta$-axis. 
4. $E$ : the expansion rate. In the self-similar expansion (Farrugia et al., 1993), $E$ is related to the time period $T_{0}$ during which the flux rope expansion had proceeded before the first encounter with the spacecraft as $E=1 / T_{0}$.

5. $s$ : the sign of the magnetic field chirality of the MC.

The set of 7 parameters listed in 1-4 above determines the duration of the MC passage by the spacecraft. Because the durations of MCs are given by observations, it can be said that there is one relationship among the 7 parameters. This relationship is utilized in the fitting procedure, as described in Appendix A. The parameter $s$ is fixed throughout the fitting procedure. The selection can be made after trying the fitting with both $s=1$ and $s=-1$.

\subsection{Torus model}

Romashets and Vandas (2003) presented the expression for the force-free magnetic field inside a toroidal magnetic cloud which is valid for any ratio of the minor radius to the major radius of the torus. We use this model with one modification to include the expansion effect. In the toroidal coordinates, $\mu, \eta$, and $\psi$ (see Romashets and Vandas, 2001, for the toroidal coordinates), the surface of a torus with the major radius $R_{M}$ and the minor radius $r_{m}$ is given by an equation $\mu=\mu_{0}$, where $\cosh \mu_{0}=R_{M} / r_{m}$. If we adopt a factor $E$ to express the expansion effect in an analogous form to the cylinder model, three magnetic field components at time $t$ are given by the following equations.

$B_{\mu}=0$

$B_{\eta}=-s B_{T} \frac{\varepsilon \cosh \mu(\cosh \mu-\cos \eta)}{2 \sinh ^{3} \mu(1+E t)^{2}} F\left(1+\tilde{\alpha}_{0}, 1+\tilde{\beta}_{0}, 2, \xi\right)$

$B_{\psi}=B_{T} \frac{\cosh \mu-\cos \eta}{\sinh \mu(1+E t)^{2}} F\left(\tilde{\alpha}_{0}, \tilde{\beta}_{0}, 1, \xi\right)$,

where $\xi=-\sinh ^{-2} \mu, F$ is the hypergeometric function,

$F(\tilde{\alpha}, \tilde{\beta}, \tilde{\gamma}, \xi)=1+\frac{\tilde{\alpha} \tilde{\beta}}{\tilde{\gamma}} \frac{\xi}{1 !}+\frac{\tilde{\alpha}(\tilde{\alpha}+1) \tilde{\beta}(\tilde{\beta}+1)}{\tilde{\gamma}(\tilde{\gamma}+1)} \frac{\xi^{2}}{2 !}+\ldots$,

and $\tilde{\alpha}_{0}=\left(1+\sqrt{1-4 \varepsilon^{2}}\right) / 4, \tilde{\beta}_{0}=\left(1-\sqrt{1-4 \varepsilon^{2}}\right) / 4$, with $\varepsilon$, the first root of $F\left(\tilde{\alpha}_{0}, \tilde{\beta}_{0}, 1,-\sinh ^{-2} \mu_{0}\right)=0 . B_{T}$ is a parameter to determine the intensity of the toroidal magnetic field. It should be noted that $B_{T}$ is different from either the field intensity along the axis of a torus or the maximum field intensity inside the torus (Romashets and Vandas, 2003).

The parameters to be determined by fitting to the torus model are briefly explained below.

1. $U_{T 0}$ and $D_{f}$ : The velocity of MC at time $t, U_{T}(t)$, is assumed to be decelerated with a deceleration factor, $D_{f}$, so that the MC speed changes in interplanetary space as:

$$
U_{T}(t)=U_{T 0}-D_{f} t
$$

The observed solar wind velocity within the MC is taken as the sum of $U_{T}(t)$ and the expansion velocity analogous to the cylinder case. The deceleration was introduced in the torus model fitting, because observed changes in the solar wind velocity cannot be reproduced by only the expansion effect.

2. $R_{M}, r_{m 0}$, and $B_{T}$ : The major radius and the minor radius at time $t=0$, and the field intensity parameter described above. The minor radius of the torus at time $t$ is given by:

$r_{m}(t)=r_{m 0}(1+E t)$

In the present fitting, we treat $R_{M}$ as a time-independent parameter. Strictly, this treatment is not self-consistent, but inclusion of expansion effects on $R_{M}$ makes the calculations much more complicated. It is hoped that this simplification does not affect the results of the analysis very much.

3. $\theta_{n}, \phi_{n}, p_{y}$, and $p_{z}$ : the latitude and longitude angles of the normal vector of the plane, defined by the axial field of the torus, and the two parameters defining the spacecraft trajectory relative to the torus axis (see Appendix B).

4. E: the expansion rate, same as for the cylinder fitting.

5. $s$ : the sign of the magnetic field chirality of the MC.

In the torus fitting, we need two parameters for defining the size of an MC, and two parameters for defining the spacecraft trajectory relative to the $\mathrm{MC}$, while one parameter is sufficient for each of them in the cylinder fitting. In addition, we introduced a new parameter, $D_{f}$, to better reproduce the velocity profiles in the torus fitting. Thus, we have a set of 10 parameters in the torus fitting, as described in 1-4, among which there is one functional relationship as in the cylinder fitting.

In the least-squares fitting process, we search for a set of parameters that provides us with the geometry of the spacecraft passage through the flank of the MC loop, such as shown that in Fig. 1a, as far as possible. The actual procedures are as follows. First, we find a set of parameters, by trial and error attempts, that yields magnetic field variations qualitatively similar to the observed variations under the conditions that (1) the equivalent local orientation of the MC axis is directed within $30^{\circ}$ from the $\mathrm{X}$-axis and (2) $|p y|$, $|p z|<1.0$. Then we execute the fitting routine starting with the parameter set as a first guess.

As a result of these procedures, we encounter two different situations. In 9 cases, the fitting routine attained convergence, yielding all parameters as well determined. For the remaining 8 cases, we found the tendency that the rms difference between the observed and calculated values became 
Table 2. Magnetic cloud parameters determined by the fitting to the torus flux rope model.

\begin{tabular}{|c|c|c|c|c|c|c|c|c|c|c|c|c|c|c|}
\hline $\begin{array}{c}\text { EVENT } \\
\text { NO. }\end{array}$ & $\begin{array}{c}R_{M}^{\mathrm{a}} \\
(\mathrm{AU})\end{array}$ & $\begin{array}{l}r_{m 0}^{\mathrm{a}} \\
(\mathrm{AU})\end{array}$ & $\begin{array}{l}\theta_{n}^{\mathrm{b}} \\
\left(^{\circ}\right)\end{array}$ & $\begin{array}{l}\phi_{n}^{\mathrm{b}} \\
\left(^{\circ}\right)\end{array}$ & $\operatorname{Sgn} B x^{\mathrm{b}}$ & $\begin{array}{c}p_{y}^{\mathrm{c}} \\
\left(r_{m 0}\right)\end{array}$ & $\begin{array}{c}p_{z}^{\mathrm{c}} \\
\left(r_{m 0}\right)\end{array}$ & $\begin{array}{c}p^{\mathrm{c}} \\
\left(r_{m 0}\right)\end{array}$ & $\begin{array}{c}B_{T}^{\mathrm{d}} \\
(\mathrm{nT})\end{array}$ & $\begin{array}{c}U_{T 0}^{\mathrm{e}} \\
(\mathrm{km} / \mathrm{s})\end{array}$ & $\begin{array}{c}D_{f}^{\mathrm{e}} \\
(\mathrm{km} / \mathrm{s} / \mathrm{h})\end{array}$ & $\begin{array}{c}H^{\mathrm{f}} \\
(\mathrm{L} / \mathrm{R})\end{array}$ & $\begin{array}{c}E \times 48^{\mathrm{g}} \\
(/ 48 \mathrm{HR})\end{array}$ & $E{ }^{\mathrm{h}} \mathrm{s}^{\mathrm{h}}$ \\
\hline 1 & 0.6 & 0.1035 & 52.0 & 230.0 & + & 3.154 & -1.985 & 0.045 & 34.6 & 344.6 & -4.66 & $\mathrm{R}$ & 0.91 & 0.237 \\
\hline 2 & 0.273 & 0.0565 & -82.3 & 337.1 & - & -0.444 & 0.639 & 0.398 & 10.7 & 401.5 & 2.03 & $\mathrm{~L}$ & -0.24 & 0.226 \\
\hline 3 & 0.6 & 0.0181 & 28.3 & 81.4 & - & -0.715 & 0.011 & 0.704 & 13.8 & 384.4 & 2.28 & $\mathrm{R}$ & -0.07 & 0.286 \\
\hline 4 & 0.399 & 0.1041 & 35.1 & 65.0 & - & 0.282 & 0.693 & 0.556 & 14.8 & 482.0 & 2.14 & $\mathrm{~L}$ & -0.09 & 0.294 \\
\hline 5 & 0.527 & 0.0255 & -69.2 & 304.2 & + & 0.720 & 0.044 & 0.620 & 29.8 & 407.2 & 1.82 & $\mathrm{~L}$ & 0.54 & 0.182 \\
\hline 6 & 0.201 & 0.0542 & -7.9 & 277.6 & + & 0.117 & 0.150 & 0.040 & 16.2 & 358.4 & 1.61 & $\mathrm{~L}$ & 0.21 & 0.113 \\
\hline 7 & 0.307 & 0.0920 & -12.4 & 50.9 & - & -0.506 & 1.194 & 0.072 & 16.7 & 348.9 & 0.71 & $\mathrm{~L}$ & 0.29 & 0.184 \\
\hline 8 & 0.6 & 0.0332 & -69.6 & 284.3 & + & -0.394 & -0.230 & 0.322 & 22.0 & 599.0 & 6.23 & $\mathrm{~L}$ & 1.49 & 0.148 \\
\hline 9 & 0.6 & 0.0532 & -45.4 & 244.7 & - & -0.494 & -0.077 & 0.489 & 14.5 & 530.9 & 5.13 & $\mathrm{~L}$ & 0.00 & 0.200 \\
\hline 10 & 0.201 & 0.0527 & 28.2 & 250.3 & + & 0.985 & -0.377 & 0.358 & 17.6 & 340.2 & 1.33 & $\mathrm{R}$ & 0.01 & 0.225 \\
\hline 11 & 0.214 & 0.0440 & -45.8 & 111.2 & + & 0.879 & -0.125 & 0.509 & 26.2 & 508.0 & 2.95 & $\mathrm{R}$ & 0.53 & 0.233 \\
\hline 12 & 0.241 & 0.0426 & -10.4 & 102.5 & + & 0.908 & -0.455 & 0.200 & 21.0 & 422.7 & 1.16 & $\mathrm{R}$ & 0.34 & 0.212 \\
\hline 13 & 0.355 & 0.0750 & 49.2 & 244.2 & - & -0.880 & -0.393 & 0.355 & 20.8 & 446.7 & 2.93 & $\mathrm{~L}$ & 0.14 & 0.170 \\
\hline 14 & 0.6 & 0.0587 & 69.3 & 36.0 & + & -0.272 & 0.702 & 0.428 & 11.8 & 451.9 & 1.17 & $\mathrm{R}$ & 0.38 & 0.269 \\
\hline 15 & 0.6 & 0.1127 & 73.3 & 341.8 & + & 0.639 & 1.484 & 0.641 & 25.8 & 496.4 & 1.61 & $\mathrm{R}$ & 0.57 & 0.154 \\
\hline 16 & 0.6 & 0.0766 & 39.4 & 81.2 & - & 0.231 & 1.058 & 0.876 & 22.3 & 561.4 & 4.81 & $\mathrm{~L}$ & 0.62 & 0.182 \\
\hline 17 & 0.6 & 0.0333 & -80.9 & 50.7 & + & 0.588 & 0.181 & 0.286 & 13.3 & 498.2 & 3.71 & $\mathrm{~L}$ & -0.51 & 0.209 \\
\hline
\end{tabular}

${ }^{a} R_{M}$ is the major radius of torus (constant), and $r_{m 0}$ is the minor radius of torus at the time of encounter.

${ }^{\mathrm{b}} \theta_{n}$ and $\phi_{n}$ are the latitude and longitude angles of a vector normal to the torus plane defined by the axial magnetic field. SgnBx indicates, by the sign of the Bx component of the axial field, on which side of the torus the spacecraft encountered.

$\mathrm{c}\left(p_{y}, p_{z}\right)$ indicates the position in the $\mathrm{YZ}$ plane of the spacecraft track from the torus axis. $p$ is the minimum distance from the torus axis to the spacecraft; all in units of $r_{m 0}$.

${ }^{\mathrm{d}} B_{T}$ is a parameter to determine the intensity of the toroidal magnetic field; see Sect. 3.2.

e $U_{T 0}$ is the velocity of MC at the time of encounter, and changes afterwards as $U_{T}(t)=U_{T 0}-D_{f} t$.

${ }^{\mathrm{f}} H$, handedness ( $\mathrm{R}$ for right-handed, $\mathrm{L}$ for left-handed).

$\mathrm{g}$ is the increase in the torus minor radius in $48 \mathrm{~h}$ as a result of self-similar expansion.

${ }^{\mathrm{h}}$ Erms is the error-estimating figure defined as the rms difference between observed and calculated fields divided by the maximum observed field intensity.

smaller and smaller with the increase in $R_{M}$, but that the fitting routine collapsed before convergence was attained. In such cases, we tried fitting with $R_{M}$ fixed, using different values in the range of $0.3-1.2 \mathrm{AU}$ (a factor 4 difference). The results of these calculations show that we obtain generally similar values for the equivalent local orientation, but obviously with changes seen in other parameters. Appendix C shows the dependence of the fitting results on the values of $R_{M}$ in more detail.

\section{Results of fitting}

We performed the model fitting with the hourly averaged data for the $17 \mathrm{MCs}$ listed in Table 1 with both cylinder and torus models, when possible. Table 2 presents the results obtained from the torus model. For $9 \mathrm{MC}$ events $R_{M}$ is presented in 3 digits. They are MC events for which $R_{M}$ could be determined by the least-squares fitting. For other MCs, we present the results obtained by taking $R_{M}=0.6 \mathrm{AU}$ as a representative value (cf. Russell et al., 2003, obtained $R m=\sim 0.8$ AU by multispacecraft observations). Listed in the table are 10 parameters described in Sect. 3.2 and 4 additional quantities, $S g n B x, p, H$, and Erms. SgnBx is the sign of the $B_{X}$ component of the axial field, indicating which side of the MC loop the spacecraft traversed (See Fig. 1 and Appendix B for more details). $p$ is the minimum distance (in $r_{m 0}$ unit) from the torus axis to the spacecraft during the passage, with the expansion effect included (Note that $p_{y}$ and $p_{z}$ are measured in the $\mathrm{YZ}$ plane, so that they do not indicate the minimum distance directly). $H$ is the handedness (right-hand or left-hand) of magnetic helicity, determined by $s(R: s=1 ; L: s=-1)$. Erms in the last column indicates the relative errors, $\Delta / B_{\max }$, where is $B_{\max }$ the maximum of the observed magnetic field intensity within the MC, and $\Delta$ is the rms deviation between the observed magnetic fields, $\boldsymbol{B}^{O}\left(t_{i}\right)$, and the model magnetic fields, $\boldsymbol{B}^{M}\left(t_{i}\right)(i=1, \ldots, N)$ :

$\Delta=\sqrt{\sum_{i}\left\{\boldsymbol{B}^{O}\left(t_{i}\right)-\boldsymbol{B}^{M}\left(t_{i}\right)\right\}^{2}} / N$

The expansion rate $E$ is presented by the amount of relative increase expected, if the expansion at a constant rate lasted 
Table 3. Magnetic cloud parameters determined by fitting to the cylinder model.

\begin{tabular}{cccccccccc}
\hline $\begin{array}{c}\text { EVENT } \\
\text { NO. }\end{array}$ & $\begin{array}{c}U_{0}^{\mathrm{a}} \\
(\mathrm{km} / \mathrm{s})\end{array}$ & $\begin{array}{c}B_{0}^{\mathrm{b}} \\
(\mathrm{nT})\end{array}$ & $\begin{array}{c}r_{0}^{\mathrm{c}} \\
(\mathrm{AU})\end{array}$ & $\begin{array}{c}\theta_{a}^{\mathrm{d}} \\
\left(^{\circ}\right)\end{array}$ & $\begin{array}{c}\phi_{a}^{\mathrm{d}} \\
\left.{ }^{\circ}\right)\end{array}$ & $\begin{array}{c}p^{\mathrm{e}} \\
\left(r_{0}\right)\end{array}$ & $\begin{array}{c}E \times 48^{\mathrm{f}} \\
(/ 48 \mathrm{~h})\end{array}$ & $\begin{array}{c}H^{\mathrm{g}} \\
(\mathrm{L} / \mathrm{R})\end{array}$ & $E r m s^{\mathrm{h}}$ \\
\hline 1 & 402.1 & 26.9 & 0.1065 & -17.0 & 308.4 & -0.156 & 0.33 & $\mathrm{R}$ & 0.246 \\
2 & 370.3 & 14.5 & 0.1536 & -8.1 & 68.8 & 0.635 & 0.22 & $\mathrm{~L}$ & 0.285 \\
3 & 350.2 & 15.5 & 0.1363 & 31.8 & 84.5 & -0.453 & 0.25 & $\mathrm{R}$ & 0.228 \\
4 & 450.0 & 16.1 & 0.1380 & 26.1 & 8.6 & 0.860 & 0.05 & $\mathrm{~L}$ & 0.304 \\
5 & 380.2 & 25.7 & 0.1063 & 53.4 & 19.8 & 0.019 & 0.50 & $\mathrm{~L}$ & 0.191 \\
6 & - & - & - & - & - & - & - & - & - \\
7 & 337.2 & 16.0 & 0.1408 & 29.3 & 76.7 & 0.484 & 0.21 & $\mathrm{~L}$ & 0.218 \\
8 & 534.2 & 24.0 & 0.1706 & 42.8 & 340.8 & -0.967 & 1.18 & $\mathrm{~L}$ & 0.264 \\
9 & 460.5 & 17.3 & 0.1316 & 35.5 & 131.3 & 0.090 & 0.50 & $\mathrm{~L}$ & 0.233 \\
10 & 319.2 & 19.1 & 0.1093 & -3.7 & 287.8 & 0.018 & 0.46 & $\mathrm{R}$ & 0.333 \\
11 & 468.3 & 24.5 & 0.1212 & -32.8 & 52.2 & 0.020 & 0.72 & $\mathrm{R}$ & 0.387 \\
12 & 408.7 & 26.8 & 0.0690 & 12.5 & 35.0 & -0.007 & 1.42 & $\mathrm{R}$ & 0.387 \\
13 & 375.8 & 23.5 & 0.1893 & -63.1 & 129.4 & 0.131 & 0.52 & $\mathrm{~L}$ & 0.335 \\
14 & 433.5 & 12.9 & 0.0126 & -3.1 & 357.6 & -0.731 & 0.44 & $\mathrm{R}$ & 0.260 \\
15 & 472.7 & 23.8 & 0.1269 & -16.3 & 322.3 & -0.712 & 0.65 & $\mathrm{R}$ & 0.163 \\
16 & 501.0 & 20.9 & 0.1057 & 38.3 & 188.6 & -0.819 & 1.36 & $\mathrm{~L}$ & 0.359 \\
17 & 435.1 & 24.0 & 0.1886 & 78.7 & 50.1 & -0.486 & 0.33 & $\mathrm{~L}$ & 0.378 \\
\hline
\end{tabular}

${ }^{\text {a }} U_{0}$ is the velocity of magnetic cloud.

${ }^{\mathrm{b}} B_{0}$ is the magnetic field intensity at the cylinder axis.

${ }^{\mathrm{c}} r_{0}$ is the radius of the magnetic cloud cylinder at the time of encounter.

${ }^{\mathrm{d}} \theta_{a}$ and $\phi_{a}$ are the latitude and longitude angles of the cylinder axis field.

e $p$ is the impact parameter.

${ }^{f} E \times 48$ is the increase in the torus minor radius in $48 \mathrm{~h}$ as a result of self-similar expansion.

g $H$ is the handedness of magnetic helicity (R for right-handed, $L$ for left-handed).

h Erms is the error-estimating figure, defined as the rms difference between the observed and calculated fields divided by the maximum observed field intensity.

for $48 \mathrm{~h}$. Table 3 shows the results of fitting with a cylinder model for the same 17 MCs.

It should be noted here that not all the values in Tables 2 and 3 are necessarily acceptable. The least-squares fitting procedure returns a set of fitted parameters regardless of whether or not they may provide calculated variations with satisfactory agreement with the observed variations. We need to examine separately which one is more plausible as the real geometries of MCs between the torus and cylinder results. In this section, we first present the results of the fitting for some representative examples, and then go on to examine which gives a more appropriate interpretation for each $\mathrm{MC}$, the torus model or the cylinder model.

\subsection{Examples}

Figure 2 shows the result of analysis for the MC encountered on 4 February 1998 (Event 6 in Table 1). Plotted from top to bottom are the magnetic field intensity, the $\mathrm{X}, \mathrm{Y}$, and $\mathrm{Z}$ components of the field in the GSE coordinate, the ratio of standard deviations to the average intensities, the solar wind speed, the number density ratio of $\mathrm{He}^{++} / \mathrm{H}^{+}$, the proton density, the proton temperature, the plasma beta based on protons, and the magnetic field vectors projected on the $\mathrm{X}-\mathrm{Y}, \mathrm{X}-\mathrm{Z}$, and $\mathrm{Y}-\mathrm{Z}$ planes. The dashed curve drawn along with the proton temperature shows the temperature statistically expected from the solar wind speed, $T_{e x}$ (Lopez, 1987). The MC boundaries are indicated by two vertical lines.

One of the conspicuous features of this MC is a very smooth, long-lasting rotation of the magnetic field vectors. A clockwise rotation of $329^{\circ}$ is seen in the Y-Z plane to proceed during a $43 \mathrm{~h}$ interval. The thick solid lines for the magnetic field and the solar wind speed depict the results of the fitting with the torus model, showing an excellent agreement with the observations. Because the angle of magnetic field rotation is so large, it is impossible to reproduce the observed magnetic field variations with such a cylinder model, as described in Sect. 3. While in a previous work, Lepping et al. (2006) invoked a new cylinder model with dual polarity to explain this type of MCs, we here restricted ourselves to a cylinder model that allows the change in the pitch angle of the magnetic field from $0^{\circ}$ at the axis to $90^{\circ}$ at the surface. For this reason, the entries for Event 6 are blank in Table 3.

The geometry of the MC encounter with the spacecraft is shown in Fig. 3, which is calculated with the fitted parameters presented in Table 2 . The figure shows the torus surface 


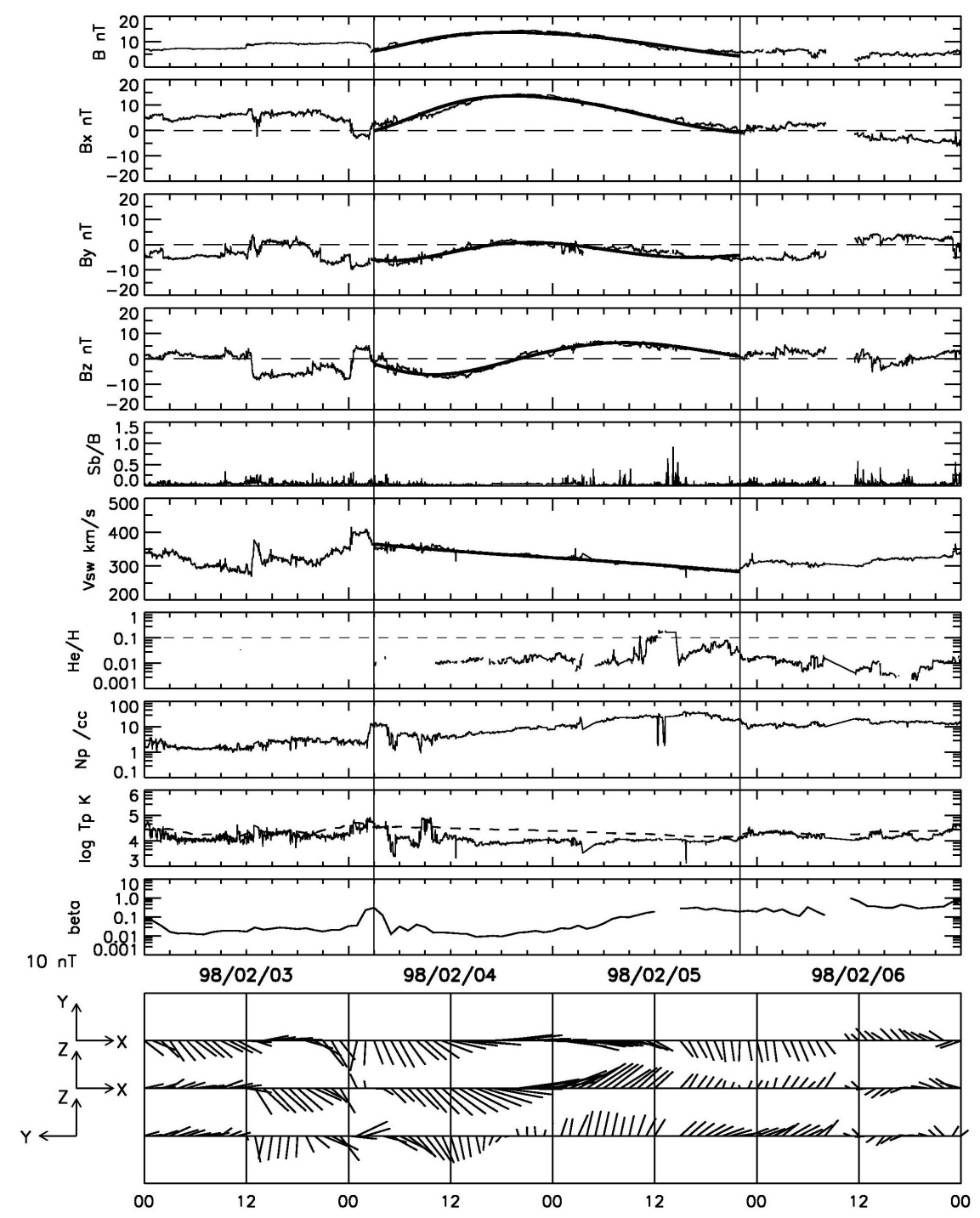

Fig. 2. Results of the torus fitting to the magnetic cloud (Event No. 6), encountered on 4 February 1998 (solid curves), are superimposed on the data plots of the observed solar wind parameters. The two vertical solid lines indicate the MC boundaries. The bottom three panels show the projected magnetic field vectors.

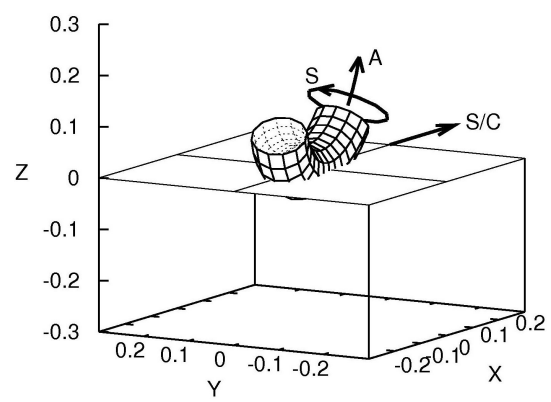

Fig. 3. Geometry of the 4 February 1998 magnetic cloud (Event No. 6) determined by fitting with the torus model. Three directions are indicated: A, axial field; S, toroidal field on the surface; and $\mathrm{S} / \mathrm{C}$, spacecraft trajectory relative to the magnetic cloud. at time $t=0$ near the solar ecliptic plane, with three arrows indicating the direction of magnetic field on the MC surface (S), the direction of the axial field of the MC (A) and the spacecraft trajectory (S/C). In this event, the spacecraft traversed the MC very close to the torus axis, almost in parallel with the axis around 19:00 UT, thus the observed magnetic fields there mostly consisted of the axial component with a very small contribution of the transverse component. Thus, we see in Fig. 2 such features as $B_{y} \approx B_{z} \approx 0$ and the reversal of $B_{z}$ around 19:00 UT.

In Fig. 2, we can see some of the characteristic features that are commonly taken as the signatures of ICMEs. They include abnormally low proton temperatures, the low plasma betas based on protons, and small fluctuations of magnetic fields. The observed proton temperature is generally low 
in comparison with that expected statistically from the solar wind speed, though the difference becomes small toward the trailing part of the MC. Similarly, the proton beta is generally low, but increases toward the trailing part in correspondence with the decrease in the magnetic field intensity and the increase in the proton number density. Though the magnetic field fluctuations are generally small throughout the 4-day period, in this case, the condition is satisfied at any rate since the fluctuations are small within the MC. The bidirectional electron heat flow event (BDE) is also evident in almost the same interval in the summary plot of the WIND 3-D Plasma and Particle Investigation (Lin et al., 1995, http://sprg.ssl.berkeley.edu/wind3dp/). However, the enhancement of $\mathrm{He}^{++}$is not clear in this MC, though we see the enhanced $\mathrm{He}^{++} / \mathrm{H}^{+}$ratio exceeding 0.1 in a restricted time interval of several hours in the trailing part of the MC.

Figure 4 presents the field and plasma data for a 4-day period, including the $\mathrm{MC}$ of Event 13, in the same format as Fig. 2. The vertical dashed line preceding the MC indicates the arrival time of a shock, which is considered to have been driven by this ICME. A striking feature with this MC is its long duration of $57 \mathrm{~h}$. Again, we can see several ICME signatures, such as the enhanced $\mathrm{He}^{++} / \mathrm{H}^{+}$ratio, the abnormally low proton temperature, the low proton plasma beta, and the small fluctuations of magnetic fields. The BDE was also observed from 18:20 UT, 19 March to 04:00 UT, 22 March.

For this MC, the model fitting was performed both with a torus model and with a cylinder model. We can see an excellent agreement between observations and results from the torus fitting, as plotted by thick solid lines. In contrast, the results from the cylinder model, shown by dotted curves, yield only a very unsatisfactory agreement with the observations. The rms difference, $\Delta$, between the observed and modeled magnetic fields is $6.5 \mathrm{nT}$, twice the rms difference from the torus fitting, $3.2 \mathrm{nT}$. Besides, it is impossible for the cylinder model to reproduce the feature that most of the magnetic field rotation takes place in the earlier half of the MC.

Figure 5 depicts the geometry of this $\mathrm{MC}$ at the time of encounter with the spacecraft in the same format as Fig. 3; Fig. 5a for the torus model, and Fig. 5b for the cylinder model. We can see two big differences between the geometries obtained from the two models. The first difference is seen in the orientations of the MC obtained from the two models. At the location where the spacecraft passed the MC, the direction of the torus axis is nearly parallel to the ecliptic plane, and is in fact nearly parallel to the $\mathrm{X}$-axis (the cone angle being $162.5^{\circ}$ : cf. Table 4 ), while the cylinder axis is highly inclined to the ecliptic plane. The angle between the torus axis and the cylinder axis is as large as $55.9^{\circ}$. The second difference is seen in the size of MC determined by the fitting. The torus fitting gives the radius of $0.0750 \mathrm{AU}$ at $t=0$, whereas the cylinder fitting gives the radius of $0.1893 \mathrm{AU}$. Because the duration of this MC is so long and the axis is so inclined from the ecliptic plane, the radius must become large in proportion to the duration in the cylinder model, whereas the radius can be much smaller for the torus model, because the curvature effect makes it possible for the spacecraft to spend a longer time, as is evident in Fig. 5a. By a close examination of the geometry of the spacecraft passage through the MC, it is seen that the spacecraft crosses the front side $(X<0)$ of the torus, close to the axis, in the earlier half of the duration, and passes just near the surface on the rear side in the latter half of the duration. Thus, the torus model explains why the magnetic field rotation of about $180^{\circ}$ was observed in the earlier half and only about $40^{\circ}$ in the latter half.

Summarizing the above results, we can say that the torus model provides a much more reasonable explanation for this MC. Though the cylinder-fitting routine returns fitted parameters, the agreement between the observed and calculated field variations is not satisfactory. This event should be taken as a case which gives us a warning when attempting interpretation of the fitting results with large rms differences, even if a qualitative agreement may be roughly attained.

Figure 6 presents the results from the torus fitting and the cylinder fitting for Event 14 in the same format as Fig. 4. Though the magnetic field intensities are rather weak throughout the MC, we can see such ICME signatures as the magnetic field rotation, the enhanced $\mathrm{He}^{++} / \mathrm{H}^{+}$ratio, the abnormally low proton temperature, and the low proton plasma beta. A very long BDE event was observed from 10 May through 11 May, along with this MC. Good agreements are obtained by both the torus fitting and the cylinder fitting, so that the difference between the two fitted curves can be hardly distiguished in this presentation. However, a close examination of the geometries obtained from two models allows us to discriminate between the two fitting results.

Figure 7 shows the geometry of the spacecraft's encounter with the MC, Fig. 7a for the torus model and Fig. $7 b$ for the cylinder model. In Table 4 we find the orientation of the cylinder MC to be very close to the $\mathrm{X}$-axis. The cone angle of the axis (defined as the angle between the cylinder axis and the $\mathrm{X}$-axis) is $3.9^{\circ}$, with the latitude and longitude angles of the axis $-3.1^{\circ}$ and $357.6^{\circ}$, respectively. Because of this geometry, the spacecraft must spend a long time within the cylinder MC once it enters the MC. As a result of this requirement, the cylinder radius was estimated to be as small as $0.0126 \mathrm{AU}$. Considering that this type of geometry should take place when the spacecraft encounters the MC near its flank, we need to take into account the curvature of the MC. The result of the torus fitting gives the local torus orientation nearly parallel to the $\mathrm{X}$-axis, and the minor radius of $0.0587 \mathrm{AU}$, if we assume $R_{M}=0.6 \mathrm{AU}$. This size is much more reasonable, being in the radius range typical to many other MCs.

Figure 8 shows the variations in the solar wind parameters observed for a 4-day period, together with the results of torus fitting (solid curve) and cylinder fitting (dotted curve) for the MC encountered on 18 April 2002 (Event 15). Two shocks are indicated by vertical dashed lines. The one preceding the MC is a shock most probably driven by this ICME and 


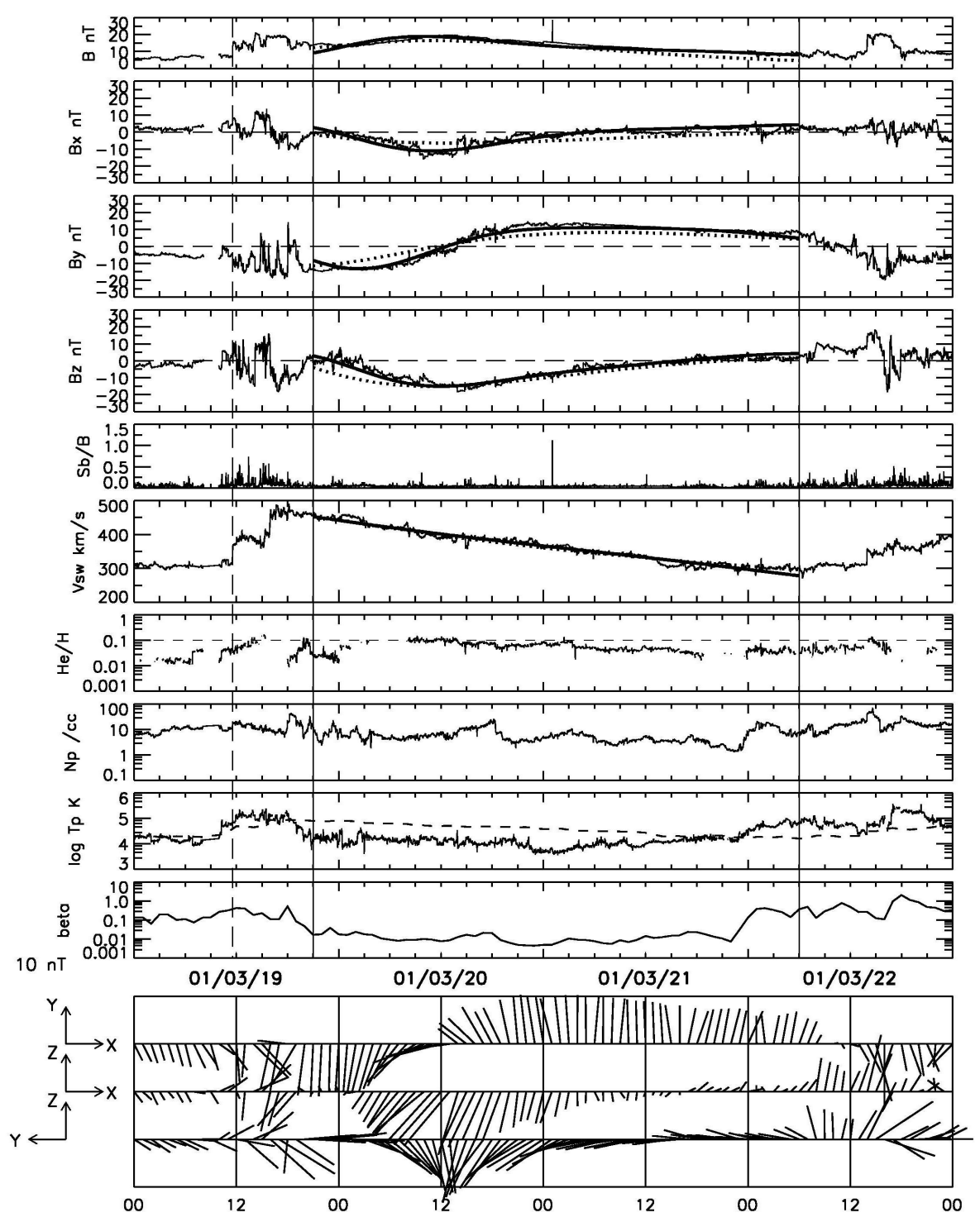

Fig. 4. Results of the fitting with the torus model (solid line) and with the cylinder model (dotted lines) to the magnetic cloud (Event No. 13) encountered on 19 March 2001 superimposed on the data plots of the observed solar wind parameters. The two vertical solid lines indicate the MC boundaries, and the vertical dashed line indicates the shock arrival time. The bottom three panels show the projected magnetic field vectors.

(a)

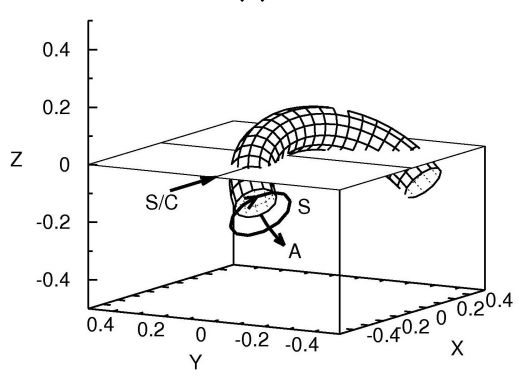

(b)

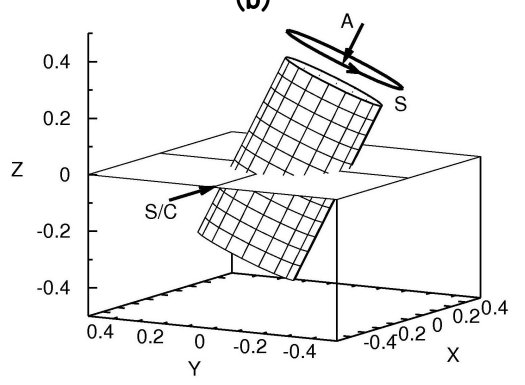

Fig. 5. Two geometries obtained for the magnetic cloud of 19 March 2001 (Event 13), from the fittings to the torus model (a) and to the cylinder model (b). Note that the result from the cylinder model is not very successful (see Fig. 4 and text). 


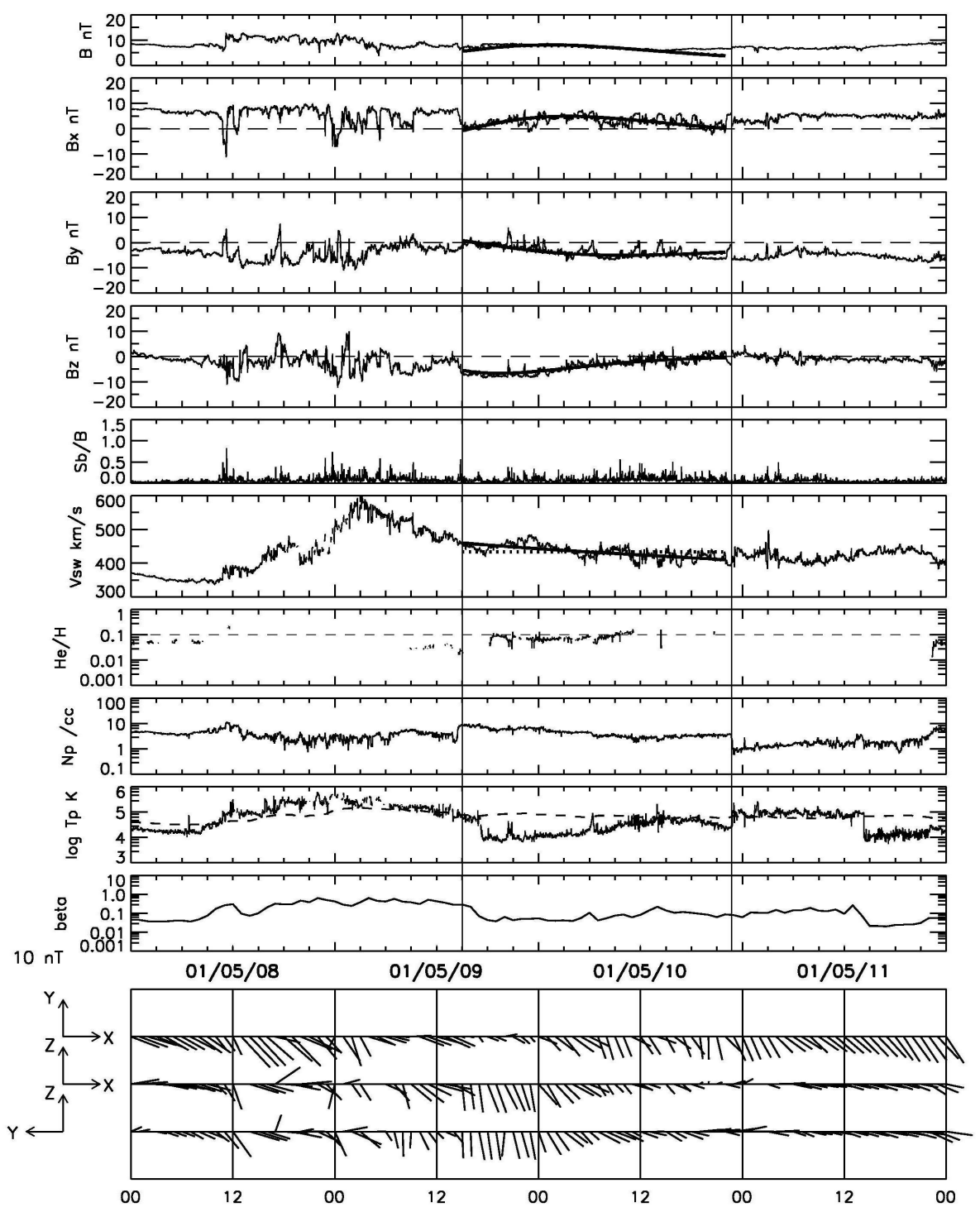

Fig. 6. Results of the fitting with the torus model (solid curve) and with the cylinder model (dotted curve) to the magnetic cloud (MC) encountered on 9 May 2001 (Event No. 14) in the same format as Fig. 4. Note that the fitting results with two models are very close to each other. The bottom three panels show the projected magnetic field vectors.

(a)

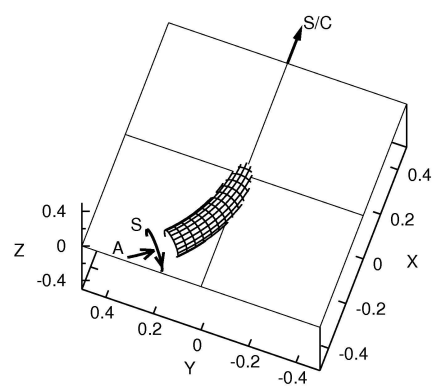

(b)

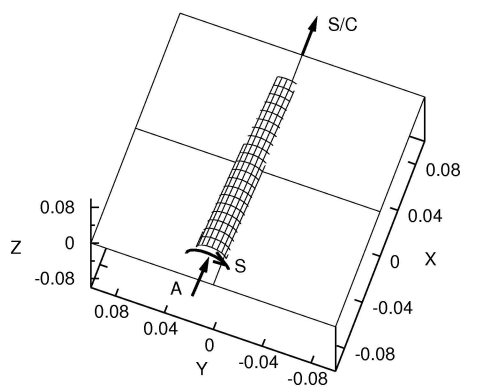

Fig. 7. Two geometries obtained for the magnetic cloud of 9 May 2001 (Event No. 14), from the fittings to the torus model (a) and to the cylinder model (b). Note that the scales are 5 times expanded in Fig. 7b. 
the other at 08:02 UT on 19 April is an internal shock which probably overtook the MC from behind. The end time of the MC was taken at 11:00 UT, 19 April, because the magnetic field rotation continued till this time, though the intensity jumped up at the shock. Again, the ICME signatures are clearly seen in the figure, such as the small fluctuations of magnetic fields, the enhanced $\mathrm{He}^{++} / \mathrm{H}^{+}$ratio, the abnormally low proton temperature, and the low proton plasma beta. The BDE was also observed throughout the MC interval.

For this MC, the fittings were performed by using only the magnetic field and solar wind velocity data from the start time of the MC to the time of the internal shock, and the remaining part was treated as if it were the interval of a data gap. This is to avoid possible errors due to the field intensity changes associated with the internal shock. The agreement between the observed and modeled variations is satisfactory for both the torus and cylinder fittings, so that the difference between the two fitted curves can hardly be recognized in this presentation.

The geometry of the MC at the time of the encounter with the spacecraft is shown in Fig. 9a for the torus fitting and in Fig. $9 \mathrm{~b}$ for the cylinder fitting. It is seen that the geometries are very similar, when viewed locally along the spacecraft trajectory, for the two fitting results in this case. A closer examination of Fig. 9a reveals that the spacecraft traverses the torus at the portion far enough from the flank so that the curvature effect becomes unimportant. Thus, it is concluded that this MC observation corresponds to the case where the spacecraft traversed rather near the apex of the MC loop than near the flank of the loop, for which both cylinder and torus models can provide reasonable interpretation of observation.

The final example is the MC encountered on 27 May 1996 (Event 2), for which the fitting results and the resultant MC geometries are shown in Figs. 10 and 11, respectively. We can see in Fig. 10 that the fitting with the cylinder model (dotted curve) is acceptable, though the torus model gives a better fitting result (solid curve). Figure 11 shows, however, that the MC geometries from two models are completely different from each other. At the location where the spacecraft passed the MC, the direction of the torus axis is nearly parallel to the $\mathrm{X}$-axis (the cone angle being $165.2^{\circ}$ ), while the cylinder axis is nearly perpendicular to the $\mathrm{X}$-axis (the cone angle being $69.0^{\circ}$ ). As a result, the angle between the torus axis and the cylinder axis is as large as $97.2^{\circ}$ (see Table 4). Besides, the flux rope sizes obtained from the two models are very different, with the radius from the torus model being $0.0565 \mathrm{AU}$, whereas the radius from the cylinder model is $0.1536 \mathrm{AU}$ at the time of the first encounter with the spacecraft.

The differences in the results from the torus and cylinder models are similar to the case that we have seen in the analysis of Event 13. More important, however, the two models provide similar magnetic field variations that are both close to the observed variation, while only the torus model pro- vides an acceptable result for the $\mathrm{MC}$ geometry in the case of Event 13. This means that two different MC geometries can explain the observations as well. One implication is that both of these geometries can take place in reality, and that the model fitting alone is insufficient in determining the real geometry for each of the particular MC observations.

\subsection{Selection of acceptable fitting results}

We further examine which model gives a more plausible geometry for each of the $17 \mathrm{MC}$ observations, the torus fitting or the cylinder fitting. For the purpose of comparison between the torus and cylinder geometries, we define the equivalent local direction of the torus axis, as is shown in Fig. 1b. The direction of the vector is selected so as to coincide approximately with the direction of the axial field, and presented by $\Theta_{e q}$ and $\Phi_{e q}$, the latitude and longitude angles. Table 4 lists the equivalent local direction of the torus axis $\Theta_{e q}$ and $\Phi_{e q}$, the cone angle of the torus axis, $(\mathrm{CA})_{e q}$, the cone angle of the cylinder axis (CA) $a$, and the angle between the cylinder axis and the torus axis, $\delta$. (The latitude and longitude angles, $\theta_{a}$, and $\phi_{a}$, are also listed again to make the comparison easier.) For those cases where the cylinder fitting results are not very good (Erms $>0.3)$, the relevant values are shown in brackets. Here, $(\mathrm{CA})_{a},(\mathrm{CA})_{e q}$, and $\delta$ are given by the following equations.

$$
\begin{aligned}
& (\mathrm{CA})_{a}=\operatorname{Cos}^{-1}\left(\cos \theta_{a} \cos \phi_{a}\right) \\
& (\mathrm{CA})_{e q}=\operatorname{Cos}^{-1}\left(\cos \Theta_{e q} \cos \Phi_{e q}\right) \\
& \delta=\operatorname{Cos}^{-1}\left(\cos \theta_{a} \cos \phi_{a} \cos \Theta_{e q} \cos \Phi_{e q}+\cos \theta_{a}\right. \\
& \left.\quad \sin \phi_{a} \cos \Theta_{e q} \sin \Phi_{e q}+\sin \theta_{a} \sin \Theta_{e q}\right)
\end{aligned}
$$

It is a noteworthy feature that in many cases the local equivalent directions of the torus axis are nearly parallel or antiparallel to the $\mathrm{X}$-axis, indicating that the spacecraft traversed the MCs apart from the loop top, closer to the loop's flank, when considered in the framework of the torus model.

By examining the fitting results in Tables 2 and 3, it is seen that the relative errors are small $(E r m s<0.3)$ for all the results from the torus model, whereas Erms $<0.3$ is satisfied for the cylinder fitting only in 9 cases. Thus, if we adopt Erms $<0.3$ as a criterion for the good agreement between the observations and the fitting results, we can classify the $17 \mathrm{MCs}$ examined into two categories: $8 \mathrm{MCs}$ for which Erms $<0.3$ is satisfied only by the fitting with a torus model (Category A), and 9 MCs for which Erms $<0.3$ is satisfied with both torus and cylinder models (Category B). Further, we divide each of these two categories into two groups, respectively. It is seen, in Category A, that the rotation angles of magnetic field vectors are larger than $180^{\circ}$ for $5 \mathrm{MCs}$, while the rotation angles are smaller than $180^{\circ}$ for the remaining 3 MCs. Thus, Category A can be divided into two groups A1 (Events 6, 11, 12, 13, and 17) and A2 (Events 4, 10, 16) by the field rotation 


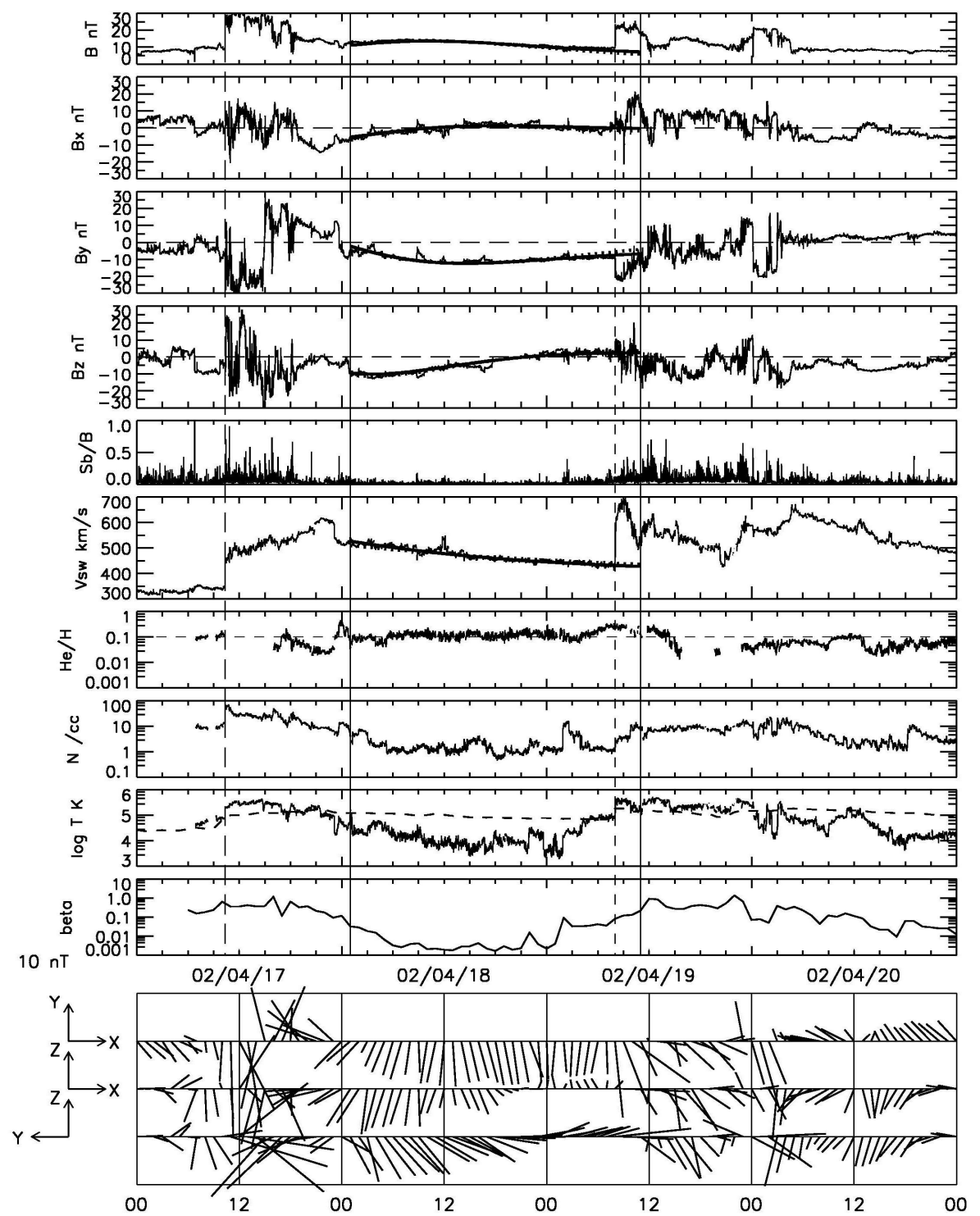

Fig. 8. Results of the fitting with the torus model (solid curve) and with the cylinder model (dotted curve) to the magnetic cloud (MC) encountered on 18 April 2002 (Event No. 15) in the same format as Fig. 4. Two shocks are indicated by two vertical dashed lines. Note that the fitting results with two models are very close to each other. The bottom three panels show the projected magnetic field vectors.

(a)

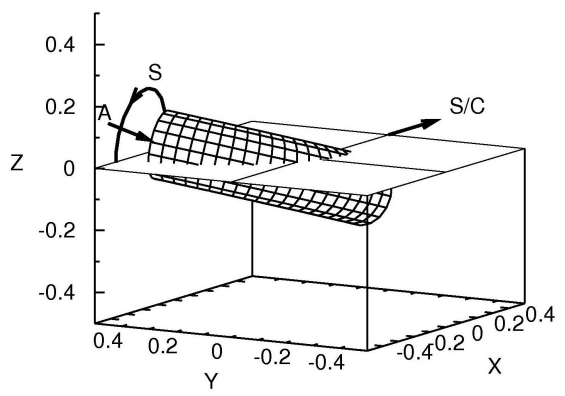

(b)

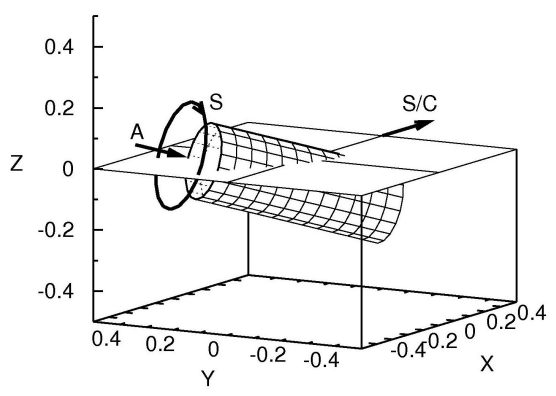

Fig. 9. Two geometries obtained for the magnetic cloud of 18 April 2002 (Event No. 15), from the fittings to the torus model (a) and to the cylinder model (b). 


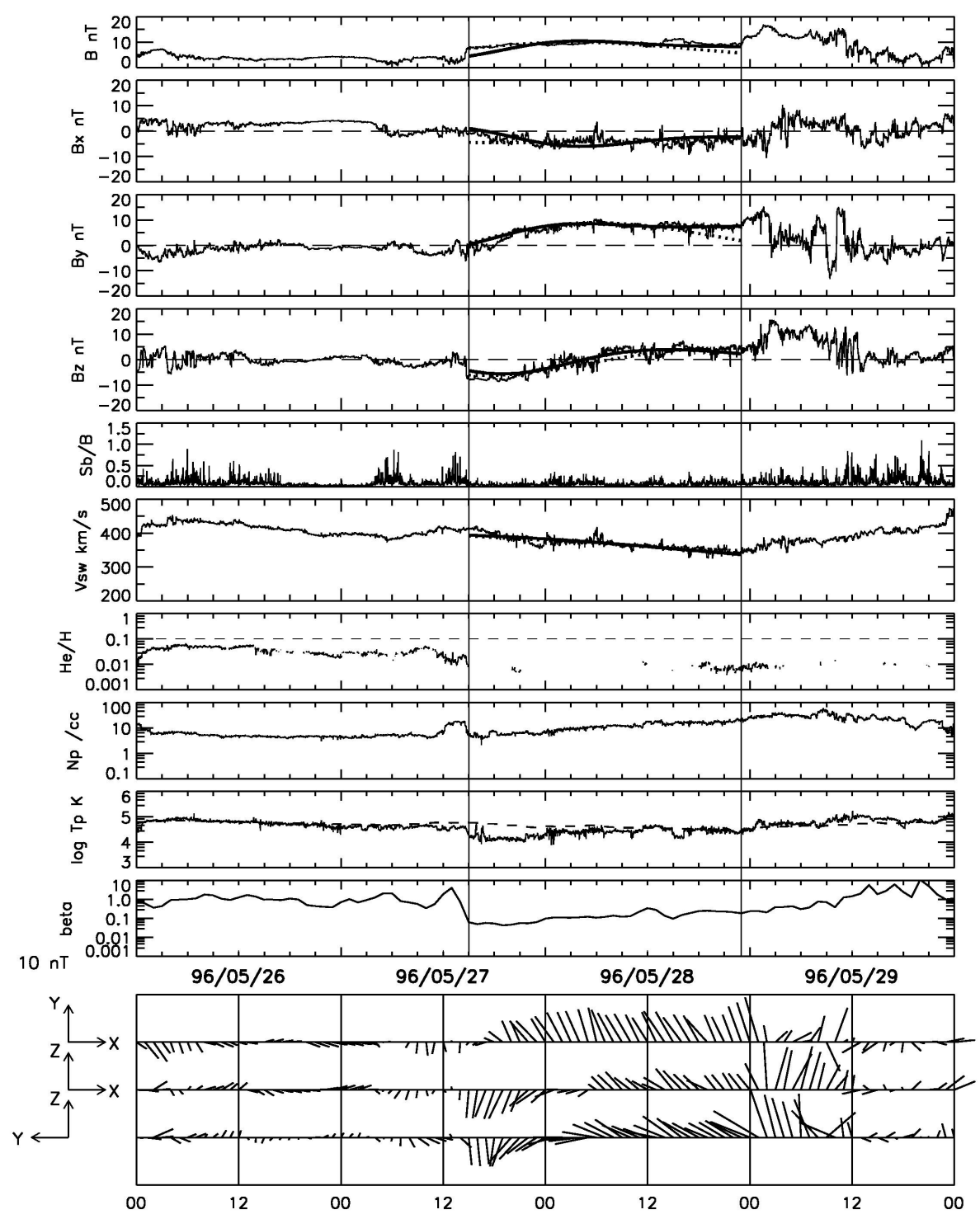

Fig. 10. Results of the fitting with the torus model (solid curve) and with the cylinder model (dotted curve) to the magnetic cloud (MC) encountered on 27 May 1996 (Event No. 2) in the same format as Fig. 4. The bottom three panels show the projected magnetic field vectors.

(a)

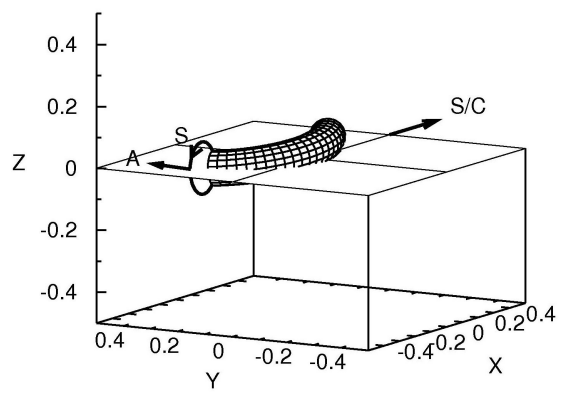

(b)

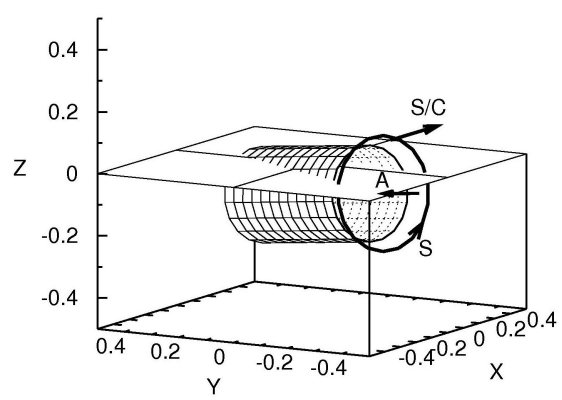

Fig. 11. Two geometries obtained for the magnetic cloud of 27 May 1996 (Event No. 2), from the fittings to the torus model (a) and to the cylinder model (b). 
Table 4. Comparison between magnetic cloud orientations obtained from the torus model and the cylinder model.

\begin{tabular}{ccccccccc}
\hline EVENT & \multicolumn{3}{c}{ TORUS FIT } & \multicolumn{3}{c}{ CYLINDER FIT } & DIF & \multirow{2}{*}{ GROUP $^{\mathrm{f}}$} \\
NO. & $\Theta_{e q}^{\mathrm{a}}$ & $\Phi_{e q}^{\mathrm{a}}$ & $(\mathrm{CA})_{e q}^{\mathrm{b}}$ & $\theta_{a}^{\mathrm{c}}$ & $\phi_{a}^{\mathrm{c}}$ & $(\mathrm{CA})_{a}^{\mathrm{d}}$ & $\delta^{\mathrm{e}}$ & \\
\hline 1 & -15.8 & 298.8 & 62.8 & -17.0 & 308.4 & 53.6 & 9.3 & $\mathrm{~B} 1$ \\
2 & -7.5 & 167.2 & 165.2 & -8.1 & 68.8 & 69.0 & 97.2 & $\mathrm{~B} 2$ \\
3 & 3.9 & 173.5 & 172.4 & 31.8 & 84.5 & 85.3 & 87.1 & $\mathrm{~B} 2$ \\
4 & 22.4 & 171.9 & 156.3 & $(26.1)$ & $(8.6)$ & $(27.4)$ & $(128.9)$ & $\mathrm{A} 2$ \\
5 & 11.5 & 1.9 & 11.7 & 53.4 & 19.8 & 55.9 & 44.3 & $\mathrm{~B} 2$ \\
6 & 1.6 & 7.4 & 7.6 & - & - & - & - & $\mathrm{A} 1$ \\
7 & 17.4 & 136.9 & 134.2 & 29.3 & 76.7 & 78.4 & 56.0 & $\mathrm{~B} 2$ \\
8 & 6.5 & 356.6 & 7.3 & 42.8 & 340.8 & 46.1 & 38.9 & $\mathrm{~B} 2$ \\
9 & 12.0 & 167.2 & 162.5 & 35.5 & 131.3 & 122.5 & 40.0 & $\mathrm{~B} 2$ \\
10 & -3.4 & 338.5 & 21.8 & $(-3.7)$ & $(287.8)$ & $(72.2)$ & $(50.6)$ & $\mathrm{A} 2$ \\
11 & -11.3 & 9.4 & 14.7 & $(-32.8)$ & $(52.2)$ & $(59.0)$ & $(44.7)$ & $\mathrm{A} 1$ \\
12 & 1.1 & 12.8 & 12.8 & $(12.5)$ & $(35.0)$ & $(36.9)$ & $(24.8)$ & $\mathrm{A} 1$ \\
13 & -16.5 & 174.3 & 162.6 & $(-63.1)$ & $(129.4)$ & $(106.7)$ & $(55.9)$ & $\mathrm{A} 1$ \\
14 & -14.8 & 350.5 & 17.5 & -3.1 & 357.6 & 3.9 & 13.6 & $\mathrm{~B} 1^{* *}$ \\
15 & -16.6 & 334.4 & 30.2 & -16.3 & 322.3 & 40.6 & 11.6 & $\mathrm{~B} 1$ \\
16 & 5.7 & 175.9 & 173.0 & $(38.3)$ & $(188.6)$ & $(140.9)$ & $(34.6)$ & $\mathrm{A} 2$ \\
17 & 6.2 & 3.7 & 7.2 & $(78.7)$ & $(50.1)$ & $(82.8)$ & $(76.1)$ & A1 \\
\hline
\end{tabular}

a The local direction of the torus axis is given by equivalent latitude and longitude angles, $\Theta_{e q}$ and $\Phi_{e q}$, respectively; see Fig. $1 \mathrm{~b}$ for definition.

$\mathrm{b}(\mathrm{CA})_{e q}$ is the cone angle of the torus direction around the X-axis; see Eq. (14).

${ }^{\mathrm{c}}$ The direction of the cylinder axis is given by $\theta_{a}$ and $\phi_{a}$; same as in Table 3 .

$\mathrm{d}(\mathrm{CA})_{a}$ is the cone angle of the cylinder axis around the X-axis; see Eq. (13).

e $\delta$ is the difference between the torus direction and the cylinder direction; see Eq. (15).

${ }^{\mathrm{f}}$ Categorization of the 17 magnetic clouds into 4 groups; A1 and A2, fitting with torus model is much better than fitting with cylinder model; B1, MCs both torus and cylinder model give similar geometry; B2 the torus and cylinder models give different geometries.

** Though this event is categorized as B1, only the torus fitting is acceptable; see Fig. 7.

angle. Category B can be divided into two groups by the difference in the orientations obtained by the torus and cylinder fittings. It is seen in Category B that $\delta$ is smaller than $30^{\circ}$ in 3 cases (Events 1, 14, and 15), whereas $\delta$ is larger than $30^{\circ}$ in the remaining 6 cases (Events 2, 3, 5, 7, 8, and 9). They constitute two groups, B1 and B2, respectively. The last column in Table 4 indicates the group to which each MC observation belongs.

The MC observations in Group A1 should be considered as cases where the spacecraft traversed the MCs deep enough to cross near the axis, and near the flank of the MC loop, almost in parallel to the torus axis, as is evident from the torus cone angles close to $0^{\circ}$ or $180^{\circ}$. A typical example has been seen with Event 6 in Figs. 2 and 3. It should be noted again that the cylinder model cannot reproduce the field rotation substantially larger than $180^{\circ}$.

For the MC events in Group A2, the torus cone angles are close to $0^{\circ}$ or $180^{\circ}$, indicating that the spacecraft traverses near the flank of MC loop, as well. In these cases, however, the spacecraft did not enter the MCs deep enough, but traversed only near the surface of the MCs, in contrast to the cases in Group A1. An additional interpretation may be desirable for Event 10, because the magnetic field rotation is large in this case compared with the other two cases. The observed solar wind field variations for this MC are compared with the result of fitting by the torus model and that by the cylinder model in Fig. 12 in the same format as Fig. 4. The most conspicuous difference between the torus and cylinder models is seen in the $B_{X}$ variation in the trailing part of the MC. Because of the relatively large angle of the magnetic field rotation, the cylinder fitting requires the spacecraft passage near the MC axis. In such geometries, it is impossible for the cylinder model to reproduce $B_{X}$ variations having different signs at the entering and exiting points. We can see in the figure that the $B_{X}$ variation is much improved by the torus model.

With regard to the MCs in Group B1, the local directions determined by the torus fitting are close to the axis directions determined by the cylinder fitting $\left(\delta<30^{\circ}\right)$. Further, we notice that the torus cone angles are relatively large for these MC events, except for Event 14. We have already seen that Event 14 is a very special case where the MC axis is nearly parallel to the $X$-axis, and therefore the torus model is required for interpreting the observation. When the torus fitting result yields the local axis orientation of a large cone angle, the curvature effect is not very significant. Therefore, the 


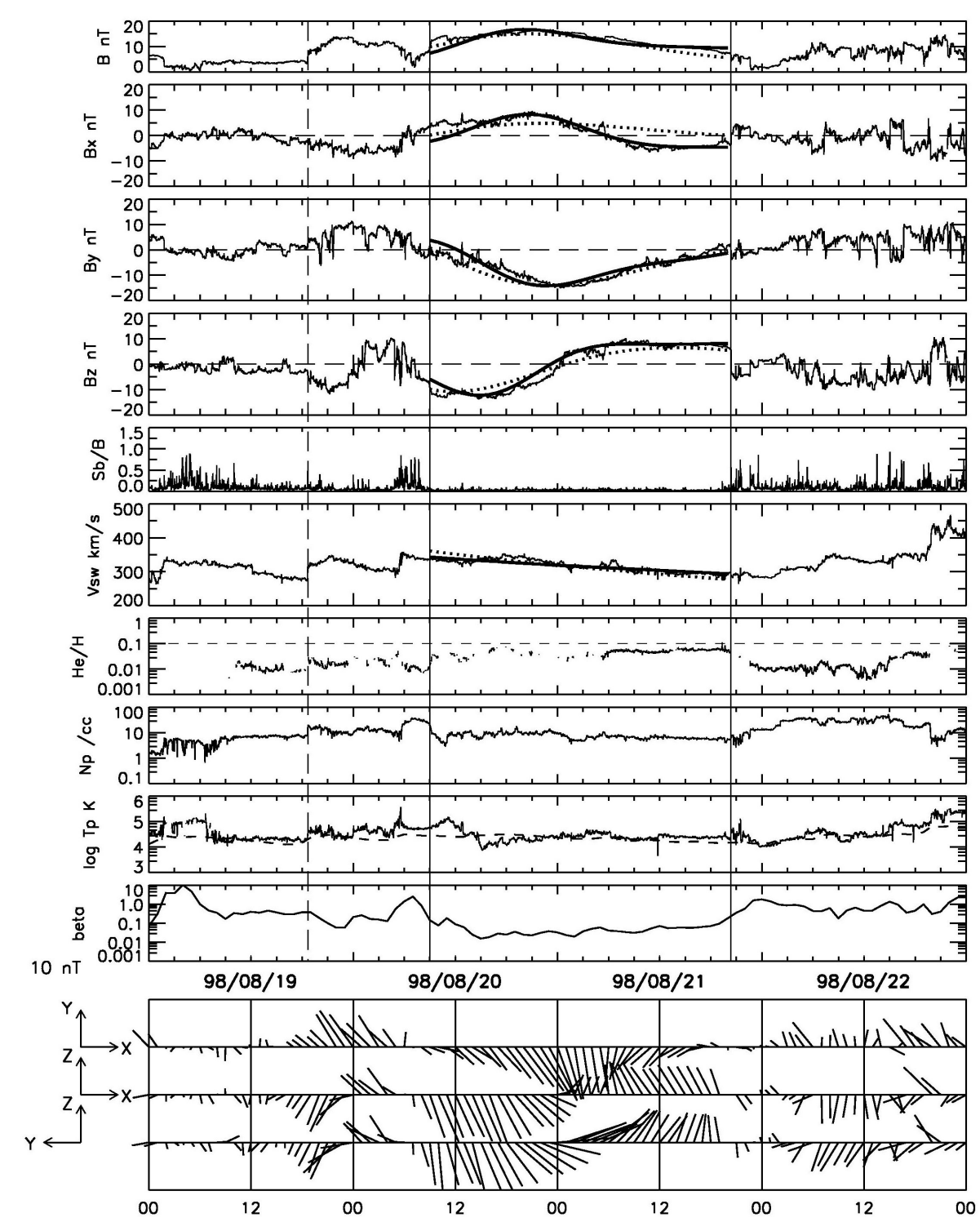

Fig. 12. Results of the fitting with the torus model (solid curve) and with the cylinder model (dotted curve) to the magnetic cloud (MC) encountered on 20 August 1998 (Event 10) in the same format as Fig. 4. The bottom three panels show the projected magnetic field vectors.

geometry of the MC encounter with the spacecraft can be approximated by the geometry of the encounter with a cylinder. This geometrical relationship takes place when the spacecraft traversed near the apex of the MC loop. The observations of 2 events in Group B1 other than Event 14 are considered to correspond to this situation. The torus fitting result in such a case is characterized by the MC geometry in which the torus is crossed by the spacecraft only on its earthward side $(X<0$ side, with $X=0$ at the center of the torus: see Appendix B for details). It is also worthwhile to point out that one of the impact parameters $\left(p_{z}\right)$ is relatively large for these 2 events. Though the torus cone angle is relatively small for Event 15 , the geometry is interpreted as a case where the spacecraft crossed the MC loop between the apex and the flank (see Figs. 8 and 9).
Finally, a new finding of the present study is that there exist MC events that can be interpreted in terms of both the torus and cylinder models, but where the fitted directions are substantially different (events in Group B2). In 2 events in this group (Events 3 and 9) for which the rotation angles of magnetic field vectors are larger than $180^{\circ}$, good agreements with the observations are obtained from the cylinder fitting, as well as from the torus fitting. This comes from the fact that magnetic field intensities are relatively small near the $\mathrm{MC}$ boundaries in the above 2 cases, so that the differences in the field directions do not make much of a contribution to the overall rms deviations. This raises the important problem that two possible geometries appear to be obtained from one observation while one definitive geometrical situation should exist in reality. One possible conclusion is that either of the 
two cases of the different geometries can take place in reality, and that the model fitting alone may not provide the definitive real geometry for any particular MC observations. It should also be noted that the results of fitting may not be very reliable when they indicate that the spacecraft traversed only near the surface of the MCs, such as for Events 8 and 15. Thus, we must admit that the analysis based on the least-squares fitting technique alone cannot always give us the right geometry of an MC.

As a possible method to determine which gives a more plausible geometry for each of the MCs, a torus model or a cylinder model, we compare the BDE characteristics with the MC geometries obtained by fitting with the two models. It is very common in BDE flux events that one of the counterstreaming fluxes is stronger than the other. The general idea for interpreting this asymmetric feature is that the suprathermal electron flux from the footpoint of the MC loop closer to the spacecraft should be stronger than that from the other footpoint of the loop. The relationship between the MC geometry and the BDE asymmetry has been examined based on this hypothesis by Phillips et al. (1992) and Kahler et al. (1999), though the results are not very definitive. If the same hypothesis is applied, it is expected that the pitch angle of the dominant electron flux should be $180^{\circ}$ for the $\mathrm{MC}$ whose axial field is toward the Sun and $0^{\circ}$ for the MC whose axial field is away from the Sun. We examine whether this relationship is satisfied or not for our $17 \mathrm{MC}$ events.

First, by comparing the cone angles determined from the torus fitting and the cylinder fitting for $6 \mathrm{MCs}$ of Group B2, we see that the axial field polarities from the two models are opposite in 3 cases (Events 2, 3, and 7). It is seen from the BDE characteristics given in Table 1 that Event 2 matches the torus model, whereas Event 3 matches the cylinder model. For Event 7, the torus model seems more favorable, though the electron heat flow is unidirectional. For the remaining 3 MCs for which both models provide the same axial field polarities, Events 8 and 9 match the hypothesis and Event 5 mismatches the hypothesis. Second, of the $3 \mathrm{MCs}$ in Group B1, the matching of the hypothesis is seen in 2 cases (Events 1 and 14). For Event 15, the hypothesis cannot be applied, because the dominant flux direction changed within the MC. Third, of the $8 \mathrm{MCs}$ in Group A1 and Group A2, the dominant flux direction changed within the MCs in 3 cases. In the remaining 5 cases we see 2 matching cases (Events 4 and 10) and 3 mismatching cases (Events 6, 12, and 13). As a whole, we see that the axial field polarity matches the direction of dominant heat flow of the BDE in only 9 out of 13 cases, excluding 4 cases of mixed heat flow polarity. If the number of cases matching the hypothesis are compared between the torus and cylinder results (with the cylinder results of Erms $>0.3$ included), the matching is seen in 8 cases (including Event 7) for the torus results, and in 7 cases for the cylinder results. Thus, one must admit that the relationship between the dominant heat flux direction and the magnetic field polarity can be violated in many cases. It is thus con- cluded that we need more careful studies on the relationship between the asymmetries in BDE fluxes and the distances from the loop footpoints. At least, temporal variations in suprathermal electron supplies at both footpoints of the MC loop must be taken into account in some cases, because there are some cases in which the dominant flux direction changes within a single MC.

Thus far, we have seen that the $17 \mathrm{MC}$ events can be divided into 4 different groups. The first group consists of the MC events of which the rotations of magnetic field are so large that it is impossible to reproduce the observed variations by the cylinder model (A1). For the second group, the cylinder model cannot satisfactorily interpret the observations, due to the important curvature effect of the MC loops, though the field rotations are less than $180^{\circ}$ (A2). The third group corresponds to the cases in which the spacecraft encountered the MC near the apex of its loop (B1). For MCs in the fourth group, though the cylinder and torus fittings both reproduce the observations satisfactorily, the resulting geometries are substantially different from each other (B2). We examined the BDE characteristics for the purpose of selecting more plausible geometries, but the results were not definitive. In order to determine the most plausible geometry of an MC, it is necessary to compare the MC characteristics obtained by the model fitting analysis with other observations relevant to the MC geometry. Here, we point out several possible observations for future studies which may provide methods for the geometry determination of the MCs. They include examining: (1) the directions of the surface normals of any discontinuities near the MC boundaries, and shock normals associated with the MCs (Jones et al., 2002; Kataoka et al., 2005), (2) multi-spacecraft observations of the same MC (Burlaga et al., 1981; Bothmer and Schwenn, 1998; Mulligan et al., 1999; Russell et al., 2003), and (3) the relationships between the magnetic field structures of MCs and the corresponding coronal magnetic structures (Marubashi, 1986, 1997; Ishibashi and Marubashi, 2004; Cremades and Bothmer, 2004).

Finally, we compare four relevant parameters obtained from the fittings with the torus and cylinder models in Fig. 13: (a) the relative fitting errors Erms, (b) the radius of the cross-sectional circle of the MC loop at time $t=0$, (c) the speed of the translational motion of the MC at $t=0$, and (d) the maximum field intensity within the MC at $t=0$. The maximum field intensities for the torus model were calculated with Eqs. (6-8), while they are given by $B_{0}$ for the cylinder model. The parameters are plotted with different marks for the above 4 groups: circles for Group A1, squares for Group A2, triangles for Group B1 (Event 14 is indicated by an asterisk), and diamonds for Group B2. Parameters for Event 6 are not plotted here, because the cylinder fitting was not performed. It should be noted that the diagrams include parameters obtained from the cylinder fitting for events of Groups A1 and A2, for which we have concluded that the torus fitting is needed. Those points should suggest caution 
a

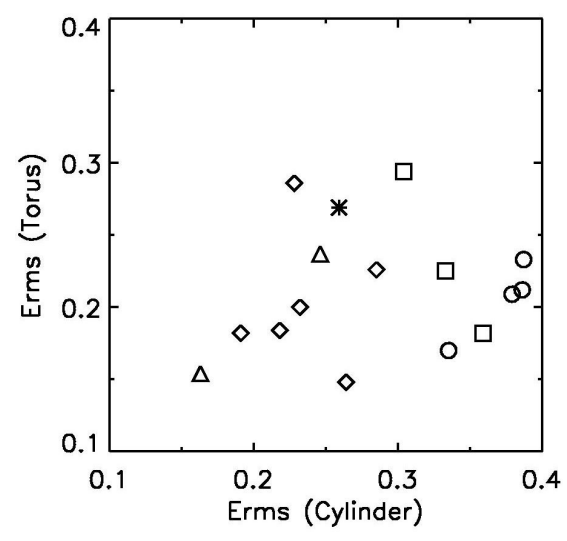

C

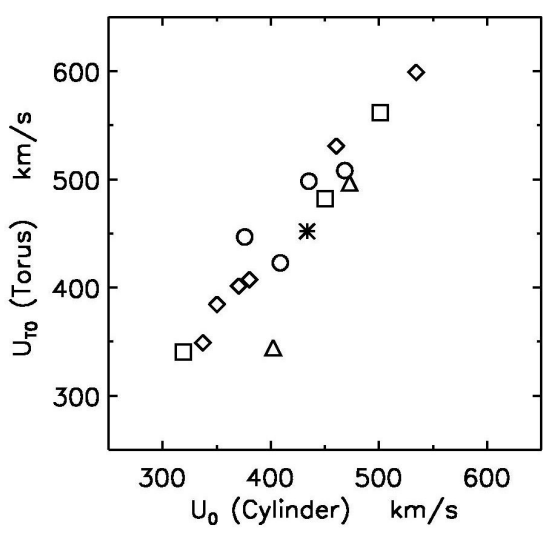

b

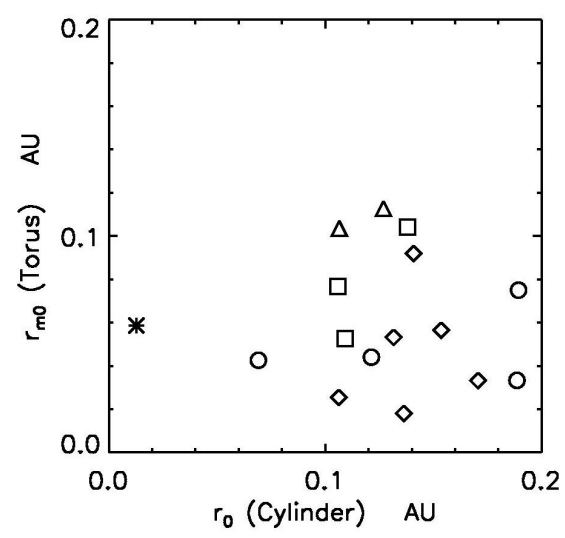

d

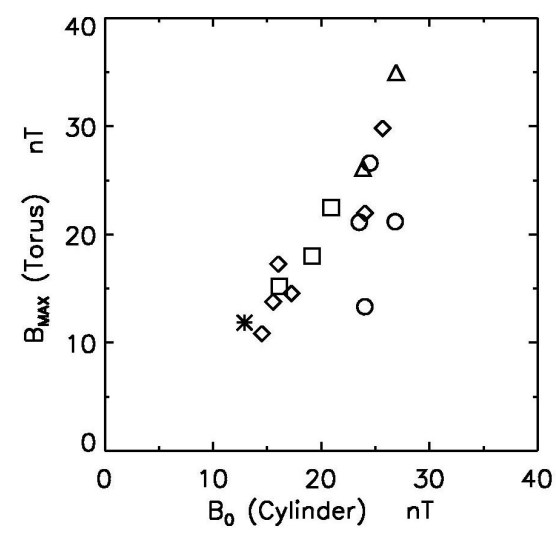

Fig. 13. Comparison of four fitted parameters obtained from the torus fitting and from the cylinder fitting: (a) the relative fitting error Erms; (b) the radius of MC flux ropes; (c) the speed of the translational motion of the MC; and (d) the maximum field intensity within the MC. Parameters for MCs in the different groups are indicated with different marks (see text).

concerning how different geometries of MCs result when the fittings with large errors were accepted. It is seen from Fig. 13a that Erms $<0.3$ is a good criterion for judging the fitting accuracy for the 17 examined MC events. Figure $13 \mathrm{~b}$ indicates that the most significant difference between the results from the two models is seen in the sizes of the MCs, while the MC speeds and the maximum field intensities from the two models are in reasonable agreement (Fig. 13c and d). As we have already seen with some examples, the radial sizes of MC flux ropes obtained with the torus model are generally smaller than those from the cylinder model. This imposes significant impacts on the estimation of magnetic flux and other related quantities carried away from the Sun by MCs (cf. Green et al., 2002; Nindos et al., 2003; Leamon et al., 2004; Lynch et al., 2005; Lepping et al., 2006).

\section{Summary}

1. We identified 17 magnetic clouds (MCs) with durations longer than $30 \mathrm{~h}$ using the solar wind data obtained from the WIND and ACE spacecraft during 10 years from 1995 through 2004. The plasma and magnetic field data for these MCs generally exhibit characteristic features commonly observed in the ICMEs, such as enhancements in the $\mathrm{He}^{++} / \mathrm{H}^{+}$ratio, bidirectional electron heat flows, abnormally low proton temperatures, and reduced magnetic field fluctuations. It should be noted, however, that all of these signatures are not necessarily seen in all of these MCs.

2. The magnetic field structures of the $17 \mathrm{MCs}$ were analyzed by the technique of the least-squares fitting to the force-free flux rope models. The analysis was made with both the cylinder and torus models when possible. The torus model was used in order to approximate the curved portion of the MCs near the flanks of the MC loops. As a result of this analysis, we have found that the $17 \mathrm{MC}$ events are classified into 4 groups; Group A1: MC events exhibiting magnetic field rotations through angles appreciably 
larger than $180^{\circ}$, which can be interpreted only by the torus model; Group A2: MC events for which the torus model is needed, because the curvature effects are important though the rotation angles are smaller than $180^{\circ}$; Group B1: MC events for which both the cylinder and torus models yield similar MC geometries with satisfactory agreements with the observed field variations; and Group B2: MC events for which results from the torus model and the cylinder model provide different geometries though good agreements with the observations are obtained from both models.

3. The 4 groups mentioned above are physically interpreted as follows. The MC observations in Group A1 are considered as cases where the spacecraft traversed near the flank of the MC loop, and deep enough to pass the axis. When the spacecraft traversed near the flank of the MC loop, but passed only near the surface, MCs in Group A2 are observed. The MC observations in Group B1 correspond to those cases where the spacecraft traversed near the apex of the MC loop, in contrast to the cases of A1 and A2. For the MC events in Group B2, the real geometry cannot be determined from only the model fitting technique, because two different geometries are possible: one near the apex, and the other near the flank of the MC loop.

4. It is far more evident that the torus model is needed for interpretation of the MCs exhibiting magnetic field rotations through angles substantially larger than $180^{\circ}$. For other cases of magnetic field variations, however, we could not find a way to foresee which model, torus or cylinder, yields a better interpretation for the observed magnetic field. Thus, for selecting an appropriate model for a given MC event, we must rely on the difference in the degree of agreement between observations and modeled results with both models. The MC for which the axis is aligned with the $\mathrm{X}$-axis is one special case which requires the torus model for interpreting the observation.

5. We found that the flux rope radii obtained from the torus fitting tend to be generally smaller than those obtained from the cylinder model. This result can be easily understood by considering the fact that the durations of a spacecraft passage through MCs tend to be relatively long when traversed near the flank of the MC loop than when traversed near the apex.

6. We tested the hypothesis that the stronger fluxes of the BDEs come from the footpoints of the MC loops closer to the spacecraft by comparing the direction of the dominant fluxes of the BDEs and the polarities of the axial field of the MCs obtained from the model fitting. As a result, we found this hypothesis is satisfied in 9 cases out of 13 cases, the matching rate being $70 \%$. This result suggests that the causes of asymmetries in BDE fluxes should be further examined with some additional possibilities taken into consideration, such as possible intensity differences between two source regions around two footpoints of the MC loops, and possible temporal variations in suprathermal electron supplies there.

\section{Conclusions and discussion}

We have presented an analysis of 17 magnetic clouds whose durations are equal to or longer than $30 \mathrm{~h}$, with torus- and cylinder-shaped flux rope models. As a result, we have obtained the following new findings:

1. There exist MC events that can be interpreted only by a torus model, corresponding to cases where the spacecraft traversed the flank of the MC loop (Group A1 and A2 in our classification).

2. There is another class of MC events for which the fittings with the torus and cylinder models yield significantly different orientations of the flux rope axis, though both models provide magnetic field variations in good agreement with observations (Group B2 in our classification).

3. The flux rope radius obtained from the torus fitting tends to be smaller than that from the cylinder fitting.

It should be pointed out that these findings impose strong impacts, in two ways at least, on our understanding of the connection between CMEs and ICMEs. First, the direction of the flux rope axis is an important factor in the understanding of the relationship between the coronal magnetic structures near the sources of CMEs and the associated ICMEs. It is plausible that one may obtain a wrong direction of the flux rope axis by using cylinder model fittings to those MCs described in 1 and 2 . We need to select the right one from two different directions obtained from two models before comparing with related coronal structures. Two MCs analyzed by Ishibashi and Marubashi (2004) and Crooker and Webb (2006), respectively, are good examples for which the torus fitting yields a better alignment between the MC axis and the orientation of the associated solar filament. Secondly, the estimation of the flux rope radius directly affects the estimation of physical quantities, such as magnetic flux and magnetic helicity carried away from the Sun by the MC (Green et al., 2002; Nindos et al., 2003; Leamon et al., 2004; Lynch et al., 2005; Lepping et al., 2006). For example, Nindos et al. (2003) showed a general tendency that for the magnetic helicity calculated from MCs to be overestimated. Such an estimate can be improved by using the smaller flux rope radius obtained from the torus model fitting.

It is thus highly desirable in future studies to determine which model gives a more realistic geometry of a given MC, the torus model or the cylinder model. Here we point out 
possible future studies which may provide methods to determine the real geometries of the MCs. They include: (a) the directions of discontinuities normals near the MC boundaries and shock normals associated with the MCs (Jones et al., 2002; Kataoka et al., 2005), (b) multi-spacecraft observations of the same MC (Burlaga et al., 1981; Bothmer and Schwenn, 1998; Mulligan et al., 1999; Russell et al., 2003).

\section{Appendix A}

\section{Magnetic fields and solar wind velocity for the cylinder model}

We derive expressions to calculate the magnetic fields and the expanding velocity within a cylindrical magnetic flux rope along the spacecraft trajectory. We use the SE coordinate system, $O_{f}-X_{f} Y_{f} Z_{f}$, moving with a flux rope with the origin $O_{f}$ taken on the flux rope axis. Let $\theta_{a}$ and $\phi_{a}$ be the latitude and longitude angles of the axial field in this coordinate system. We make a new coordinate system $O_{f}-X_{1} Y_{1} Z_{1}$ by rotating $O_{f}-X_{f} Y_{f} Z_{f}$ around the $X_{f}$-axis, so that the $X_{1}-Y_{1}$ plane contains the flux rope axis. This transformation can be made by adopting the rotation angle $\Theta$ satisfying the following equations.

$$
\left\{\begin{array}{l}
\cos \Theta=\cos \theta_{a} \sin \phi_{a} / \sqrt{\sin ^{2} \theta_{a}+\cos ^{2} \theta_{a} \sin ^{2} \phi_{a}} \\
\sin \Theta=\sin \theta_{a} / \sqrt{\sin ^{2} \theta_{a}+\cos ^{2} \theta_{a} \sin ^{2} \phi_{a}}
\end{array}\right.
$$

As a result of this transformation, the flux rope axis is toward the longitude angle $\Phi$ in the $X_{1}-Y_{1}$ plane, where $\Phi$ is given by

$$
\left\{\begin{array}{l}
\cos \Phi=\cos \theta_{a} \cos \phi_{a} \\
\sin \Phi=\sqrt{\sin ^{2} \theta_{a}+\cos ^{2} \theta_{a} \sin ^{2} \phi_{a}}
\end{array}\right.
$$

Suppose that the spacecraft enters the flux rope at time $t=0$ and exits at $t=t_{d}$ (i.e. $t_{d}$ is the duration of MC passage), and let $\left\{X_{1}(t), Y_{1}(t), Z_{1}(t)\right\}$ be the position of the spacecraft at time $t\left(0 \leq t \leq t_{d}\right)$. Then it follows

$$
\left\{\begin{array}{l}
X_{1}(t)=X_{1}(0)+U_{0} t \\
Y_{1}(t)=Y_{1}(0)=0 \\
Z_{1}(t)=Z_{1}(0)=r_{0} p
\end{array}\right.
$$

The radial distance from the cylinder axis to the spacecraft at time $t, \rho(t)$, is given by

$$
\rho^{2}(t)=X_{1}^{2}(t) \sin ^{2} \Phi+Y_{1}^{2}(t)+Z_{1}^{2}(t)
$$

If we define an angle $\beta$, the elevation angle of the spacecraft position from the $X_{1}-Y_{1}$ plane measured on the plane perpendicular to the cylinder axis, it is given by

$$
\left\{\begin{array}{l}
\cos \beta=-X_{1}(t) \sin \Phi / \rho(t) \\
\sin \beta=Z_{1}(t) / \rho(t)
\end{array}\right.
$$

Using the angles $\beta$ and $\Phi$ defined above, the expansion speed of the MC cylinder and the magnetic field at the spacecraft position at time $t$ are given as follows.

$$
\begin{aligned}
& \left\{\begin{array}{l}
v_{X 1}=-v_{\rho} \cos \beta \sin \Phi \\
v_{Y 1}=v_{\rho} \cos \beta \cos \Phi \\
v_{Z 1}=v_{\rho} \sin \beta
\end{array}\right. \\
& \left\{\begin{array}{l}
B_{X 1}=B_{\zeta} \cos \Phi+B_{\varphi} \sin \beta \sin \Phi \\
B_{Y 1}=B_{\zeta} \sin \Phi-B_{\varphi} \sin \beta \cos \Phi, \\
B_{Z 1}=B_{\varphi} \cos \beta
\end{array}\right.
\end{aligned}
$$

where $v_{X 1}, v_{Y 1}, v_{Z 1}$ and $B_{X 1}, B_{Y 1}, B_{Z 1}$ are the velocity and magnetic field components in the $O-X_{1} Y_{1} Z_{1}$ system, and $v_{\rho}, B_{\phi}, B_{\zeta}$ are given by Eqs. (2), (4), and (5) in the text, respectively.

Finally, we obtain the velocity and magnetic field components in the GSE coordinate system, $v_{X}, v_{Y}, v_{Z}$ and $B_{X}, B_{Y}$, $B_{Z}$, by rotation of $O_{f}-X_{1} Y_{1} Z_{1}$ around the $X_{1}$-axis through the angle $-\Theta$ as follows.

$$
\begin{aligned}
& \left\{\begin{array}{l}
v_{X}=-v_{\rho} \cos \beta \sin \Phi-U_{0} \\
v_{Y}=v_{Y 1} \cos \Theta-v_{Z 1} \sin \Theta \\
v_{Z}=v_{Y 1} \sin \Theta+v_{Z 1} \cos \Theta
\end{array}\right. \\
& \left\{\begin{array}{l}
B_{X}=B_{X 1} \\
B_{Y}=B_{Y 1} \cos \Theta-B_{Z 1} \sin \Theta \\
B_{Z}=B_{Y 1} \sin \Theta+B_{Z 1} \cos \Theta
\end{array}\right.
\end{aligned}
$$

In Eq. (A8), the motion of the flux rope relative to the spacecraft is taken into account. In the actual model fitting, we used the speed of the solar wind, $V_{S W}$, instead of $v_{X}, v_{Y}, v_{Z}$.

$$
V_{S W}=\sqrt{v_{X}^{2}+v_{Y}^{2}+v_{Z}^{2}}
$$

Equations (A10) and (A9) together with Eq. (A3) present variations of the solar wind speed and the magnetic field that should be observed by the spacecraft when traversing the flux rope cylinder.

In the actual calculation, we further need to determine $X_{1}(0)$ in Eq. (A3). Noting that the spacecraft is on the surface of the cylinder at times, $t=0$ and $t=t_{d}$, we obtain from Eq. (A4):

$$
\begin{aligned}
& r_{0}^{2}=X_{1}^{2}(0) \sin ^{2} \Phi+Y_{1}^{2}(0)+Z_{1}^{2}(0) \\
& r_{0}^{2}\left(1+E t_{d}\right)^{2}=\left(X_{1}(0)+U_{0} t_{d}\right)^{2} \sin ^{2} \Phi \\
&+Y_{1}^{2}\left(t_{d}\right)+Z_{1}^{2}\left(t_{d}\right)
\end{aligned}
$$

Simple manipulation of these equations yields $X_{1}(0)$ and an expression connecting the 7 fitting parameters, as given below.

$$
\begin{aligned}
& X_{1}(0)=-r_{0} \sqrt{1-p^{2}} / \sin \Phi \\
& E=\left(\sqrt{\left(X_{1}(0)+U_{0} t_{d}\right)^{2} \sin ^{2} \Phi+Y_{1}^{2}(0)+Z_{1}^{2}(0)} / r_{0}-1\right) / t_{d}
\end{aligned}
$$




\section{Appendix B}

\section{Magnetic fields and solar wind velocity for the torus model}

We derive expressions to calculate the magnetic fields and the expanding velocity within a torus-shaped magnetic flux rope along the spacecraft trajectory. We first define the SE coordinate system moving with the torus, with the origin taken at the center of a circle drawn by the axial field of the torus, $O$-XYZ. Let $\theta_{n}$ and $\phi_{n}$ be the latitude and longitude angles of a vector normal to the plane containing the axial field in this coordinate system. To make the calculations easier, we transform $\mathrm{O}-\mathrm{XYZ}$ to the torus-referred Cartesian coordinate, $O-X_{T} Y_{T} Z_{T}$, in which the plane of the torus axis is contained in the $X_{T}-Y_{T}$ plane, and the $Z_{T}$ axis is contained in the $X-Z$ plane. This transformation can be made by first defining $O-X_{1} Y_{1} Z_{1}$ by rotation of $O-X Y Z$ around the Xaxis through angle $\Theta$, and then rotating $O-X_{1} Y_{1} Z_{1}$ around $Y_{1}$-axis through angle $\Phi$, where $\Theta$ and $\Phi$ are given by the following equations.

$$
\begin{aligned}
& \left\{\begin{array}{l}
\sin \Theta=-\cos \theta_{n} \sin \phi_{n} / \sqrt{\sin ^{2} \theta_{n}+\cos ^{2} \theta_{n} \sin ^{2} \phi_{n}} \\
\cos \Theta=\sin \theta_{n} / \sqrt{\sin ^{2} \theta_{n}+\cos ^{2} \theta_{n} \sin ^{2} \phi_{n}}
\end{array}\right. \\
& \left\{\begin{array}{l}
\sin \Phi=\cos \theta_{n} \cos \phi_{n} \\
\cos \Phi=\sqrt{\sin ^{2} \theta_{n}+\cos ^{2} \theta_{n} \sin ^{2} \phi}
\end{array}\right.
\end{aligned}
$$

The expansion velocity of the torus flux rope can be easily calculated by invoking two additional coordinate systems: a cylinder coordinate system $(R, \Psi, h)$ and a toroidal cylinder system $(\rho, \beta, \varsigma)$, which are related to the $O-X_{T} Y_{T} Z_{T}$ system as follows.

$R=\sqrt{X_{T}^{2}+Y_{T}^{2}}, \quad \Psi=\operatorname{Tan}^{-1}\left(Y_{T} / X_{T}\right), \quad h=Z_{T}$

$\rho \cos \beta=R-R_{M}, \quad \rho \sin \beta=h, \quad \zeta=-R \Psi$.

The expansion velocity given by Eq. (2) in the text can be expressed with these coordinate systems as

$V_{\rho}=v_{\rho}, \quad V_{\beta}=0, \quad V_{\zeta}=0$

$V_{R}=V_{\rho} \cos \beta, \quad V_{\Psi}=0, \quad V_{h}=V_{\rho} \sin \beta$.

Then, we obtain three components of the expansion velocity in the $O-X_{T} Y_{T} Z_{T}$ coordinate system as follows.

$$
\left\{\begin{array}{l}
V_{X T}=v_{\rho} \cos \beta \cos \Psi \\
V_{Y T}=v_{\rho} \cos \beta \sin \Psi \\
V_{Z T}=v_{\rho} \sin \beta
\end{array}\right.
$$

Now, we calculate the magnetic field given by Eqs. (6-8) in the text as a function of $X_{T}, Y_{T}$, and $Z_{T}$. The toroidal coordinates $\mu, \eta$, and $\varphi$ are determined by the following relationships with $X_{T}, Y_{T}$, and $Z_{T}$ (Romashets and Vandas, 2003).

$$
\left\{\begin{array}{l}
X_{T}=a \sinh \mu \cos \varphi /(\cosh \mu-\cos \eta) \\
Y_{T}=a \sinh \mu \sin \varphi /(\cosh \mu-\cos \eta) \\
Z_{T}=a \sin \eta /(\cosh \mu-\cos \mu)
\end{array} .\right.
$$

The backward relationships are

$$
\left\{\begin{array}{c}
\cosh \mu=\left(X_{T}^{2}+Y_{T}^{2}+Z_{T}^{2}+a^{2}\right) / \\
\sqrt{\left(X_{T}^{2}+Y_{T}^{2}+Z_{T}^{2}+a^{2}\right)^{2}-4 a^{2}\left(X_{T}^{2}+Y_{T}^{2}\right)} \\
\cos \eta=\left(X_{T}^{2}+Y_{T}^{2}+Z_{T}^{2}-a^{2}\right) / \\
\sqrt{\left(X_{T}^{2}+Y_{T}^{2}+Z_{T}^{2}-a^{2}\right)^{2}+4 a^{2} Z_{T}^{2}} \\
\tan \varphi=Y_{T} / X_{T}
\end{array}\right.
$$

In this coordinate system, the equation $\mu=\mu_{0}$ defines a toroid with the major and minor radii $R_{M}$ and $r_{m}$, which are related to $a$ and $\mu_{0}$ as follows.

$a=\sqrt{R_{M}^{2}-r_{m}^{2}}$

$R_{M} / r_{m}=\cosh \mu_{0}$

$a / r_{m}=\sinh \mu_{0}$.

Manipulating Eq. (B8), we obtain

$$
\left[\begin{array}{l}
d X_{T} \\
d Y_{T} \\
d Z_{T}
\end{array}\right]=\left(w_{i j}\right)\left[\begin{array}{l}
h_{\mu} d \mu \\
h_{\eta} d \eta \\
h_{\varphi} d \varphi
\end{array}\right],
$$

where $h_{\mu}=h_{\eta}=a /(\cosh \mu-\cos \eta)$,

$h_{\varphi}=a \sinh \mu /(\cosh \mu-\cos \eta)$ are the Lamè coefficients, and $\left(w_{i j}\right)$ is a matrix, of which each element is given as follows.

$w_{11}=\frac{1-\cosh \mu \cos \eta}{\cosh \mu-\cos \eta} \cdot \cos \varphi$,
$w_{12}=-\frac{\sinh \mu \sin \eta}{\cosh \mu-\cos \eta} \cdot \cos \varphi$,
$w_{13}=-\sin \varphi$

$w_{21}=\frac{1-\cosh \mu \cos \eta}{\cosh \mu-\cos \eta} \cdot \sin \varphi$,

$w_{22}=-\frac{\sinh \mu \sin \eta}{\cosh \mu-\cos \eta} \cdot \sin \varphi$,

$w_{23}=\cos \varphi$

$w_{31}=-\frac{\sinh \mu \sin \eta}{\cosh \mu-\cos \eta}$,

$w_{32}=-\frac{1-\cosh \mu \cos \eta}{\cosh \mu-\cos \eta}$,

$w_{33}=0$. 

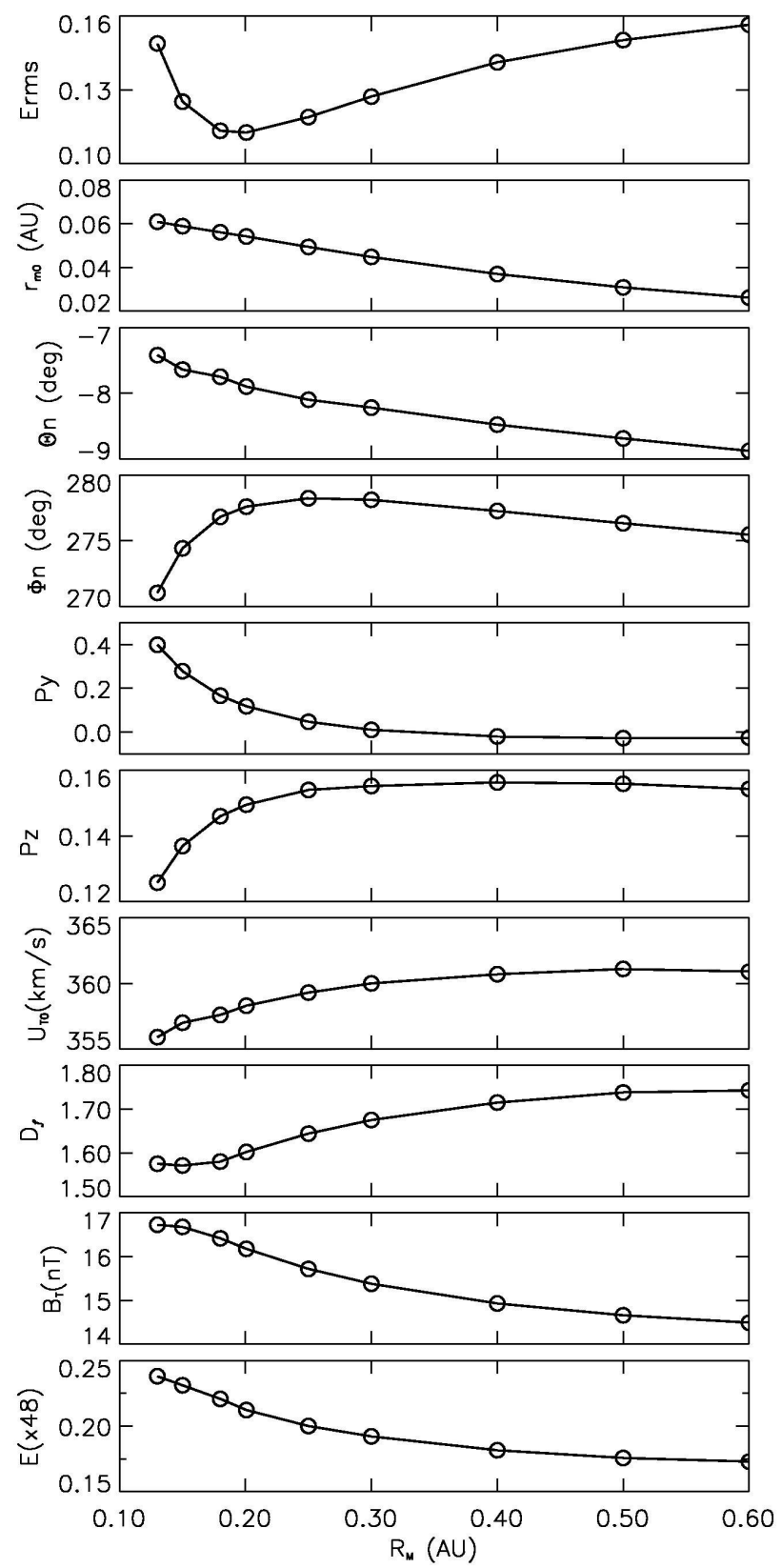

Fig. C1. Dependence of the torus parameters obtained for the magnetic cloud of 4 February 1998 (Event No. 6), on the change of the major radius $R_{M}$.

Thus, for the magnetic field vector $\left(B_{\mu}, B_{\eta}, B_{\varphi}\right)$ in the toroidal coordinate system, which is given in Sect. 3.2, the Cartesian components $B_{X T}, B_{Y T}, B_{Z T}$ in the $O-X_{T} Y_{T} Z_{T}$ coordinate system are given by

$$
\left[\begin{array}{l}
B_{X T} \\
B_{Y T} \\
B_{Z T}
\end{array}\right]=\left(w_{i j}\right)\left[\begin{array}{l}
B_{\mu} \\
B_{\eta} \\
B_{\varphi}
\end{array}\right]
$$

Then, we present expressions to calculate the spacecraft trajectory within the torus as a function of time from $t=0$ to $t=t_{d}$, which is needed to calculate the magnetic field and the expansion velocity to be measured by the spacecraft. Let $\left\{X_{1}(t), Y_{1}(t), Z_{1}(t)\right\}$ be the position of the spacecraft in the $O-X_{1} Y_{1} Z_{1}$ system at time $t$, then it follows

$$
\left\{\begin{array}{l}
X_{1}(t)=X_{1}(0)+U_{T 0} t-(1 / 2) D_{f} t^{2} \\
Y_{1}(t)=Y_{1}(0)=Y_{10}=\mp\left(\operatorname{sgn}\left(B_{X}\right)\right) R_{M}+r_{m 0} p_{y} \\
Z_{1}(t)=Z_{1}(0)=Z_{10}=r_{m 0} p_{z}
\end{array}\right.
$$

The $Y_{1}-Z_{1}$ plane cuts the torus on both sides of $Y_{1}>0$ and $Y_{1}<0$, where $B_{X 1}<0$ and $B_{X 1}>0$, respectively. We must select an appropriate one of the two $Y_{10}$ values corresponding to the side on which the spacecraft pass the torus. By defining $Y_{10}$ in this way, $p_{y}$ gives the distance normalized by $r_{m 0}$ from the torus axis to the spacecraft measured on the $Y_{1}-Z_{1}$ plane.

Finally, we calculate $X_{1}(0)$ and the expansion rate of the torus, $E$, in a similar way to the case of a cylinder model (Appendix A). The satellite position in the $O-X_{T} Y_{T} Z_{T}$ coordinate system is given by

$$
\left\{\begin{array}{l}
X_{T}(t)=X_{1}(t) \cos \Phi-Z_{10} \sin \Phi \\
Y_{T}(t)=Y_{10} \\
Z_{T}(t)=X_{1}(t) \sin \Phi+Z_{10} \cos \Phi
\end{array}\right.
$$

Noting that $\left\{X_{T}(0), Y_{T}(0), Z_{T}(0)\right\}$ and $\left\{X_{T}\left(t_{d}\right), Y_{T}\left(t_{d}\right)\right.$, $Z_{T}\left(t_{d}\right)$ \}are both on the torus surface, we obtain

$$
\begin{aligned}
& \left(\sqrt{X_{T}^{2}(0)+Y_{T}^{2}(0)}-R_{M}^{2}\right)^{2}+Z_{T}^{2}(0)=r_{m 0}^{2} \\
& \left(\sqrt{X_{T}^{2}\left(t_{d}\right)+Y_{T}^{2}\left(t_{d}\right)}-R_{M}^{2}\right)^{2}+Z_{T}^{2}\left(t_{d}\right)=r_{m 0}^{2}\left(1+E t_{d}\right)^{2} .
\end{aligned}
$$

Equation (B20) is ascribed to the 4th-degree equation for $X_{T}(0)$, substitution of the minimum root of which into Eq. (B19) gives $X_{1}(0)$. Now that $X_{1}(0)$ is obtained, the lefthand side of Eq. (B21) is known. If we express this quantity by LHS, the expansion rate is given by

$$
E=\left(\sqrt{\mathrm{LHS}} / r_{m 0}-1\right) / t_{d}
$$

\section{Appendix C}

\section{Effects of changing $R_{M}$ on the torus fitting}

Figure $\mathrm{C} 1$ depicts how the fitting result changes depending on the selection of the major radius of the torus, $R_{M}$, for the MC encountered on 4 February 1998 (Event 6). We see that the best fit is obtained for $R_{M}=0.2$ and that the adjustment takes place in other parameters by changing $R_{M}$ and that the relative rms errors of the fitting are sufficiently small throughout the range of the changing $R_{M}$ in this particular case. It should be especially noted that the orientation of the 
Table C1. Magnetic cloud parameters for $R_{M}=0.3 \mathrm{AU}$, and for $R_{M}=1.2 \mathrm{AU}$.

\begin{tabular}{|c|c|c|c|c|c|c|c|c|c|c|c|c|}
\hline $\begin{array}{c}\text { EVENT } \\
\text { NO. }\end{array}$ & $\begin{array}{c}R_{M}^{\mathrm{a}} \\
(\mathrm{AU})\end{array}$ & $\begin{array}{c}r_{m 0}^{\mathrm{a}} \\
(\mathrm{AU})\end{array}$ & $\begin{array}{l}\theta_{n}^{\mathrm{b}} \\
\left(^{\circ}\right)\end{array}$ & $\begin{array}{l}\phi_{n}^{\mathrm{b}} \\
\left(^{\circ}\right)\end{array}$ & $\begin{array}{c}p_{y}^{\mathrm{c}} \\
\left(r_{m 0}\right)\end{array}$ & $\begin{array}{c}p_{z}^{\mathrm{c}} \\
\left(r_{m 0}\right)\end{array}$ & $\begin{array}{c}p^{c} \\
\left(r_{m 0}\right)\end{array}$ & $\begin{array}{c}B_{T}^{\mathrm{d}} \\
(\mathrm{nT})\end{array}$ & $\begin{array}{c}U_{T 0}^{\mathrm{e}} \\
(\mathrm{km} / \mathrm{s})\end{array}$ & $\begin{array}{c}D_{f}^{\mathrm{e}} \\
(\mathrm{km} / \mathrm{s} / \mathrm{h})\end{array}$ & $\begin{array}{l}E \times 48^{\mathrm{f}} \\
(/ 48 \mathrm{~h})\end{array}$ & $\operatorname{Erms}^{g}$ \\
\hline \multirow{2}{*}{$1^{*}$} & 0.3 & 0.0919 & 51.4 & 230.0 & 1.895 & -1.089 & 0.075 & 41.6 & 328.0 & -6.40 & 1.35 & 0.232 \\
\hline & 1.2 & 0.1145 & 59.3 & 230.0 & -3.206 & 6.469 & 0.036 & 30.8 & 354.7 & -3.76 & 0.64 & 0.244 \\
\hline \multirow[b]{2}{*}{3} & 0.3 & 0.0289 & 27.5 & 77.2 & -0.708 & 0.015 & 0.636 & 12.1 & 383.7 & 2.29 & -0.12 & 0.330 \\
\hline & 1.2 & 0.0135 & 31.9 & 83.2 & -0.702 & -0.008 & 0.699 & 13.8 & 385.4 & 2.33 & 0.02 & 0.274 \\
\hline \multirow[b]{2}{*}{8} & 0.3 & 0.0526 & -80.4 & 304.3 & -0.323 & -0.068 & 0.245 & 23.1 & 586.5 & 5.31 & 1.40 & 0.163 \\
\hline & 1.2 & 0.0178 & -62.1 & 276.6 & -0.363 & -0.326 & 0.322 & 21.7 & 604.7 & 6.67 & 1.55 & 0.142 \\
\hline \multirow{2}{*}{9} & 0.3 & 0.0583 & -48.2 & 243.5 & -0.547 & -0.061 & 0.514 & 12.5 & 530.9 & 5.18 & -0.14 & 0.275 \\
\hline & 1.2 & 0.0401 & -59.3 & 246.8 & -0.578 & -0.365 & 0.501 & 14.6 & 531.3 & 5.12 & -0.09 & 0.168 \\
\hline \multirow{2}{*}{14} & 0.3 & 0.0783 & 56.5 & 35.4 & -0.144 & 0.471 & 0.233 & 12.4 & 443.9 & 0.62 & 0.42 & 0.278 \\
\hline & 1.2 & 0.0427 & 68.0 & 57.4 & -0.435 & 0.680 & 0.522 & 11.9 & 455.0 & 1.38 & 0.38 & 0.266 \\
\hline \multirow{2}{*}{$15^{*}$} & 0.3 & 0.1073 & 70.5 & 341.8 & 0.468 & 1.191 & 0.573 & 28.5 & 493.3 & 1.39 & 0.66 & 0.189 \\
\hline & 1.2 & 0.1152 & 75.2 & 341.8 & 1.203 & 1.998 & 0.669 & 24.3 & 499.1 & 1.83 & 0.50 & 0.148 \\
\hline \multirow{2}{*}{16} & 0.3 & 0.1296 & 75.8 & 16.9 & 0.736 & 0.410 & 0.737 & 31.0 & 546.3 & 3.70 & 0.53 & 0.190 \\
\hline & 1.2 & 0.0529 & 40.3 & 83.7 & 0.291 & 1.101 & 0.904 & 22.0 & 563.5 & 4.99 & 0.65 & 0.182 \\
\hline \multirow{2}{*}{17} & 0.3 & 0.0547 & -80.9 & 330.3 & 0.587 & 0.238 & 0.122 & 12.7 & 502.3 & 4.03 & -0.48 & 0.265 \\
\hline & 1.2 & 0.0178 & -78.8 & 73.0 & 0.608 & 0.156 & 0.361 & 14.0 & 495.9 & 3.56 & -0.50 & 0.192 \\
\hline
\end{tabular}

${ }^{\text {a }} R_{M}$, major radius of torus; $r_{m 0}$, minor radius at the time of encounter.

$\mathrm{b}$ The latitude $\left(\theta_{n}\right)$ and longitude $\left(\phi_{n}\right)$ angles of a vector normal to the torus plane.

$\mathrm{c}\left(p_{y}, p_{z}\right)$, the position of the spacecraft track on the YZ plane, and $p$, the closest approach distance to the axis.

${ }^{\mathrm{d}} B_{T}$, a parameter determining the magnetic field intensity; see Sect. 3.2.

e $U_{T 0}$, the velocity of $\mathrm{MC}$ at the time of encounter, and $D_{f}$, the deceleration factor; see Eq. (10).

${ }^{\mathrm{f}}$ Increase in the torus minor radius in $48 \mathrm{~h}$ as a result of expansion.

$\mathrm{g}$ The error estimating figure.

* For these MC events, fitting was made with both $R_{M}$ and $\phi_{n}$ fixed.

fitted torus is maintained in the range within $10^{\circ}$. It is also seen that the minor radius $r_{m 0}$ decreases with an increase in $R_{M}$. This change comes from the requirement that the spacecraft should traverse the MC during the same time interval with similar speeds.

For $8 \mathrm{MC}$ events, as described in Sect. 3, the fitting routine collapsed before the convergence could be attained, when $R_{M}$ was included as one of the free parameters to be determined by the least-squares method. Therefore, we calculated other parameters with a modified fitting routine with $R_{M}$ values fixed in the range of $0.3-1.2 \mathrm{AU}$. Through this procedure, we found the magnetic field variations calculated from the torus model becomes less sensitive to $R_{M}$ change for large $R_{M}$ values. This tendency may explain the reason why the fitting routine collapsed in those cases when $R_{M}$ is included as a free parameter.

Table $\mathrm{C} 1$ presents the parameters obtained for $8 \mathrm{MCs}$ from the torus fitting with fixed $R_{M}$ values of 0.3 and $1.2 \mathrm{AU}$. For these $8 \mathrm{MCs}$, the fitting routine could not attain convergence when $R_{M}$ was included as one of the free parameters (see the last part of Sect. 3). The minor radius $r_{m 0}$ decreases with an increase in $R_{M}$, as has been seen in Fig. $\mathrm{C} 1$, where the rate of change in $r_{m 0}$ is generally smaller than the rate of change in $R_{M}$. It is also seen that the changes in the torus plane direction $\left(\theta_{n}\right.$ and $\left.\phi_{n}\right)$ are generally small for changes in $R_{M}$, in the range of $0.3-1.2 \mathrm{AU}$. Though the changes in the torus plane direction are not small for Events 16 and 17 in this range, we can see, by comparing with Table 2, that the changes are small in the $R_{M}$ range of $0.6-1.2 \mathrm{AU}$. The fittings for Events 1 and 15 need a special comment. Because these cases involved a spacecraft passage near the apex of the MC loop, the torus model of any direction can yield similar field variations, in as much as the local orientations of the torus at the spacecraft passage are similar. Therefore, we executed the fitting routine with one parameter $\left(\phi_{n}\right)$ fixed to be consistent with the value obtained for $R_{M}=0.6 \mathrm{AU}$. 
Acknowledgements. We thank the WIND SWE, and 3DP teams for providing the WIND data. Authors' thankfulness is directed to NSSDC from which some parts of the WIND solar wind data were retrieved through NASA's SPDF. We also thank ACE MAG and SWEPAM instrument teams and the ACE Science Center for providing the ACE data. We also thank A. Szabo for WIND/MFI data support. This work was partly supported by the project, Development of Korean Space Weather Center, of KASI.

Topical Editor R. Forsyth thanks two anonymous referees for their help in evaluating this paper.

\section{References}

Borrini, G., Gosling, J. T., Bame, S. J., and Feldman, W. C.: Helium abundance enhancements in the solar wind, J. Geophys. Res., 87, 7370-7378, 1982.

Bothmer, V. and Schwenn, R.: The structure and origin of magnetic clouds in the solar wind, Ann. Geophys., 16, 1-24, 1998, http://www.ann-geophys.net/16/1/1998/.

Burlaga, L. F.: Magnetic clouds and force-free fields with constant alpha, J. Geophys. Res., 93, 7217-7224, 1988.

Burlaga, L. F., Sittler, E., Mariani, F., and Schwenn, R.: Magnetic loop behind an interplanetary shock, J. Geophys. Res., 86, 66736684, 1981.

Burlaga, L. F., Lepping, R. P., and Jones, J. A.: Global configuration of a magnetic cloud, in: Physics of Magnetic Flux Ropes, Geophys. Monogr. Ser., vol.58, edited by: Russell, C. T., Priest, E. R., and Lee, L. C., AGU, Washington, D.C., 373-377, 1990.

Cremades, H. and Bothmer, V.: On the three-dimensional configuration of coronal mass ejections, Astron. Astrophys., 422, 307322, 2004.

Collier, M. R., Lepping, R. P., and Berdichevsky, D.: A statistical study of interplanetary shocks and pressure pulses internal to magnetic clouds, J. Geophys. Res., 112, A06102, doi:10.1029/2006JA011714, 2007.

Crooker, N. U. and Webb, D. F.: Remote sensing of the solar site of interchange reconnection associated with the May 1997 magnetic cloud, J. Geophys. Res., 111, A08108, doi:10.1029/2006JA011649, 2006.

Dungey, J. W.: Interplanetary magnetic field and the auroral zones, Phys. Rev. Lett., 6, 47-48, 1961.

Farrugia, C. J., Burlaga, L. F., Osherovich, V. A., and Lepping, R. P.: A comparative study of dynamically expanding forcefree, constant-alpha magnetic configurations with applications to magnetic clouds, in: Solar Wind Seven, edited by: Marsch, E. and Schwenn, R., COSPAR, vol. 3, Pergamon, New York, 611614, 1992.

Farrugia, C. J., Burlaga, L. F., Osherovich, V. A., Richardson, I. G., Freeman, M. P., Lepping, R. P., and Lazarus, A. J.: A study of an expanding interplanetary magnetic cloud and its interaction with the Earth's magnetosphere: The interplanetary aspect, J. Geophys. Res., 98, 7621-7632, 1993.

Goldstein, H.: On the field configuration in magnetic clouds, in: Solar Wind Five, edited by: Neugebauer, M., NASA Conf. Publ., 2280, 731-733, 1983.

Gosling, J. T.: Coronal mass ejections and magnetic flux ropes in interplanetary space, in: Physics of Magnetic Flux Ropes, Geophys. Monogr. Ser., vol.58, edited by: Russell, C. T., Priest, E. R., and Lee, L. C., AGU, Washington, D.C., 343-364, 1990.
Gosling, J. T., Pizzo, V., and Bame, S. J.: Anomalously low proton temperatures in the solar wind following interplanetary shock waves: Evidence for magnetic bottles?, J. Geophys. Res., 78, 2001-2009, 1973.

Gosling, J. T., Baker, D. N., Bame, S. J., Feldman, W. C., Zwickl, R. D., and Smith, E. J.: Bidirectional solar wind electron heat flux events, J. Geophys. Res., 92, 8519-8535, 1987.

Green, L. M., López Fuentes, M. C., Mandrini, C. H., Démoulin, P., Van Driel-Gesztelyi, L., and Culhane, J. L.: The magnetic helicity budget of a CME-prolific active region, Solar Phys., 208, 43-68, 2002.

Hirshberg, J. S., Bame, S. J., and Robbins, D. E.: Solar flares and solar helium enrichments: July 1965-July 1967, Sol. Phys., 23, 467-486, 1972.

Ishibashi, H. and Marubahsi, K.: Structure of interplanetary magnetic cloud on April 16, 1999 and its origin estimated by fitting the torus-shaped flux rope model, Geophys. Res. Lett, 31, L21807, doi:10.1029/2004GL020702, 2004.

Jones, G. H., Rees, A., Balogh, A., and Forsyth, R. J.: The draping of heliospheric magnetic fields upstream of coronal mass ejecta, Geophys. Res. Lett., 29, 1520, doi:10.1029/2001GL014110, 2002

Kahler, S. W., Crooker, N. U., Gosling, J. T.: A magnetic polarity and chilarity analysis of ISEE 3 interplanetary magnetic clouds, J. Geophys. Res., 104, 9911-9918, 1999.

Kataoka, R., Watari, S., Shimada, N., Shimazu, H., and Marubashi, K.: Downstream structures of interplanetary fast shocks associated with coronal mass ejections, Geophys. Res. Lett., 32, L12103, doi:10.1029/2005GL022777, 2005.

Leamon, R. J., Canfield, R. C., Jones, S. L., Lambkin, K., Lundberg, B. J., and Pevtsov, A. A.: Helicity of magnetic clouds and their associated active regions, J. Geophys. Res., 109, A05106, doi:10.1029/2003JA010324, 2004.

Lepping, R. P., Jones, J. A., and Burlaga, L. F.: Magnetic field structure of interplanetary magnetic cloud, J. Geophys. Res., 95, 11957-11 965, 1990.

Lepping, R. P., Acuna, M. H., Burlaga, L. F., Farrell, W. M., Slavin, J. A., Schatten, K. H., Mariani, F., Ness, N. F., Neubauer, F. M., Whang, Y. C., Byrnes, J. B., Kennon, R. S., Panetta, P. V., Sheifele, J., and Worley, E. M.: The Wind magnetic field investigation, Space Sci. Rev., 71, 207-229, 1995.

Lepping, R. P., Berdichevsky, D. B., Wu, C.-C., Szabo, A., Narock, T., Mariani, F., Lazarus, A. J., and Quivers, A. J.: A summary of WIND magnetic clouds for years 1995-2003: Model-fitted parameters, associated errors, and classifications, Ann. Geophys., 24, 215-245, 2006, http://www.ann-geophys.net/24/215/2006/.

Lin, R. P., Anderson, K. A., Ashford, S., Carlson, C., Curtis, D., Ergun, R., Larson, D., McFadden, J., McCarthy, M., Parks, G. K., Rème, H., Bosqued, J. M., Coultelier, J., Cotin, F., D’Uston, C., Wenzel, K.-P., Sanderson, T. R., Henrion, J., Ronnet, J. C., and Paschmann, G.: A three-dimensional plasma and energetic particle investigation for the Wind spacecraft, Space Sci. Rev., 71, 125-153, 1995.

Lopez, R.: Solar cycle invariance in solar wind proton temperature relationships, J. Geophys. Res., 92, 11 189-11 194, 1987.

Lynch, B. J., Zurbuchen, T. H., Fisk, L. A., and Antiochos, S. K.: Internal structure of magnetic clouds: Plasma and composition, J. Geophys. Res., 108, 1239, doi:10.1029/2002JA009591, 2003. 
Lynch, B. J., Gruesbeck, J. R., Zurbuchen, T. H., and Antiochos, S. K.: Solar cycle-dependent helicity transport by magnetic clouds, J. Geophys. Res., 110, A08107, doi:10.1029/2005JA011137, 2005.

Marubashi, K.: Structure of the interplanetary magnetic clouds and their solar origins, Adv. Space Res., 6(6), 335-338, 1986.

Marubashi, K.: The space weather forecast program, Sapce Sci. Rev., 51, 197-214, 1989.

Marubashi, K.: Interplanetary magnetic flux ropes and solar filaments, in: Coronal Mass Ejections, Geophys. Monogr. Ser., vol. 99, edited by: Crooker, N., Joselyn, J. A., and Feynman, J., AGU, Washington, D.C., 147-156, 1997.

Marubashi, K.: Physics of interplanetary magnetic flux ropes: Toward prediction of geomagnetic storms, Adv. Space Res., 26(1), 55-66, 2000.

Marubashi, K.: Interplanetary magnetic flux ropes, J. Comm. Res. Lab., 49(3), 41-59, 2002.

McComas, D. J., Bame, S. J., Barker, P., Feldman, W. C., Philips, J. L., Riley, P., and Griffee, J. W.: Solar wind electron proton alpha monitor (SWEPAM) for the advanced composition explorer, Space Sci. Rev., 86, 563-612, 1998.

Mulligan, T., Russell, C. T., and Luhman, J. G.: Solar cycle evolution of the structure of magnetic clouds in the inner heliosphere, Geophys. Res. Lett., 25, 2959-2962, 1998.

Mulligan, T., Russell, C. T., Elliot, D., Gosling, J. T., and Luhman, J. G.: Inversion studies of magnetic cloud structure at $0.7 \mathrm{AU}$ : Solar cycle variation, Geophys. Res. Lett., 28, 891-894, 2001.

Mulligan, T., Russell, C. T., Anderson, B. J., Lohr, D. A., Rust, D., Toth, B. A., Zanetti, L. J., Acuna, M. H., Lepping, R. P., and Gosling, J. T.: Intercomparison of NEAR and Wind interplanetary coronal mass ejection observations, J. Geophys. Res., 104, 28 217-28 223, 1999.

Neugebauer, M. and Goldstein, R.: Particle and field signatures of coronal mass ejections in the solar wind, in: Coronal Mass Ejections, Geophys. Monogr. Ser., vol. 99, edited by: Crooker, N., Joselyn, J. A., and Feynman, J., AGU, Washington, D.C., 245252, 1997.

Nindos, A., Zhang, J., and Zhang, H.: The magnetic helicity budget of solar active regions and coronal mass ejections, Astrophys. J., 594, 1033-1048, 2003.

Ogilvie, K. W., Chornay, D. J., Fitzenreiter, R. J., Hunsaker, F., Keller, J., Lobell, J., Miller, G., Scudder, D., Sittler Jr., E. C., Torbert, R. B., Bodet, D., Needell, G., Lazarus, A. J., Steinberg, J. T., Tappan, J. H., Mavretic, A., and Gergin, E.: SWE, a comprehensive plasma instrument for the WIND spacecraft, Space Sci. Rev., 71, 55-77, 1995.
Phillips, J. L., Gosling, J. T., McComas, D. J., Bame, S. J., and Feldman, W. C.: Quantitative analysis of bidirectional electron fluxes within coronal mass ejections at $1 \mathrm{AU}$, in: Solar Wind Seven, edited by: Marsch, E. and Schwenn, R., Pergamon, Tarrytown, N. Y., 651-656, 1992.

Richardson, I. G. and Cane, H. V.: Regions of abnormally low proton temperature in the solar wind (1963-1991) and their association with ejecta, J. Geophys. Res., 100, 23 397-23 412, 1995.

Richardson, I. G. and Cane, H. V.: Identification of interplanetary coronal mass ejections at $1 \mathrm{AU}$ using multiple solar wind plasma composition anomalies, J. Geophys. Res., 109, A09104, doi:10.1029/2004JA010598, 2004.

Romashets, R. P. and Vandas, M.: Dynamics of a toroidal magnetic cloud in the solar wind, J. Geophys. Res., 106, 10615-10624, 2001.

Romashets, R. P. and Vandas, M.: Force-free field inside a toroidal magnetic cloud, Geophys. Res. Lett., 30(20), 2065, doi:10.1029/2003GL017692, 2003.

Russell, C. T., Mulligan, T., and Anderson, B. J.: Radial variation of magnetic flux ropes: Case studies with ACE and NEAR, in: Solar Wind Ten, edited by: Velli, M., Bruno, R., and Malara, F., AIP, 121-124, 2003.

Shimazu, H. and Marubashi, K: New method for detecting interplanetary flux ropes, J. Geophys. Res., 105, 2365-2373, 2000.

Shimazu, H. and Vandas, M.: A self-similar solution of expanding cylindrical flux ropes for any polytropic index values, Earth, Planets and Space, 54, 783-790, 2002.

Smith, C. W., L'Heureux, W. J., Ness, N. F., Acuna, M. H., Burlaga, L. F., and Scheifele, J.: The ACE magnetic fields experiment, Space Sci. Rev., 86, 613-632, 1998.

Tsurutani, B. T. and Gonzalez, W. D.: The interplanetary causes of magnetic storms: A review, in: Magnetic Storms, Geophys. Monogr. Ser., vol.98, edited by; Tsurutani, B. T., Gonzalez, W. D., Kamide, Y., and Arballo, J. K., AGU, Washington, D.C., 7789, 1997.

Vandas, M. and Geranios, A.: November 17-18, 1975, event: A clue to an internal structure of magnetic clouds?, J. Geophys. Res., 106, 1849-1858, 2001.

Vandas, M. and Romashets, R. P.: A force-free field with constant alpha in an oblate cylinder: A generalization of the Lundquist solution, Astron. Astrophys., 398, 801-807, 2003.

Zhao, X. P., Hoeksema, J. T., and Marubashi, K.: Magnetic cloud Bs events and their dependence on cloud parameters, J. Geophys. Res., 106, 15 643-15 656, 2001. 